\title{
Sequence Designs for Interference Mitigation in Multi-Cell Networks
}

\author{
Ying-Che Hung, Sheng-Yuan Peng, and Shang-Ho (Lawrence) Tsai, Senior Member, IEEE
}

\begin{abstract}
We propose a training sequence that can be used at the handshaking stage for multi-cell networks. The proposed sequence is theoretically proved to enjoy several nice properties including constant amplitude, zero autocorrelation, and orthogonality in multipath channels. Moreover, the analytical results show that the proposed sequence can greatly reduce the multi-cell interference (MCI) induced by carrier frequency offset (CFO) to a negligible level. Therefore, the CFO estimation algorithms designed for single-user or single-cell environments can be slightly modified, and applied in multi-cell environments; an example is given for showing how to modify the estimation algorithms. Consequently, the computational complexity can be dramatically reduced. Simulation results show that the proposed sequences and the CFO estimation algorithms outperform conventional schemes in multi-cell environments.
\end{abstract}

Index Terms-Sequence designs, multi-cell interference (MCI), synchronization, OFDM, CFO estimation.

\section{INTRODUCTION}

$\mathbf{R}$ ECENTLY, OFDM based two-tier cell networks, which consist of a conventional macrocell cellular network overlaid with small cells access points (FAPs), attract extensive research attention due to their enhanced capacity and improved coverage, see e.g., [1]-[3]. In many cases, different cells may appear in the same frequency band to increase user capacity and data capacity. One example is to deploy multiple cells in a band and use spatial multiplexing to avoid multicell and multi-user interference [4]-[6]. Another example is in cooperative networks [7]-[9], where one user may receive and combine the signals from several cells to increase data capacity. However, in both examples, multi-cell interference (MCI) appears at the early handshaking stage and degrades the performance of cell ID (identity) detection, synchronization, and channel estimation. More specifically, in the first example, spatial multiplexing may be applied after the handshaking stage because it needs channel information. In the second example, the user needs to detect cell IDs first, and then determine proper cells to cooperate. Thus, how to attain a smooth handshaking under the appearance of MCI is an important issue.

One way is via mitigating the MCI. There are several interference mitigation methods including power control and

Manuscript received April 22, 2013; revised September 3, 2013; accepted November 5, 2013. The associate editor coordinating the review of this paper and approving it for publication was S. Bhashyam.

This work is supported by the National Science Council (NSC), Taiwan, R.O.C. Cooperative Agreement No. 99-2221-E-009-100-MY3 and 102-2221E009-017-MY3.

The authors are with the Department of Electrical Engineering, National Chiao Tung University, Hsinchu, Taiwan (e-mail: yingjhe.ece95g@g2.nctu.edu.tw, shanghot@alumni.usc.edu).

Digital Object Identifier 10.1109/TWC.2013.120413.130703 spectrum allocation [10]-[13]. For instance, the optimal decentralized spectrum allocation technology was proposed in [10], and a distributed cell power control mechanism to achieve higher signal-to-interference plus noise ratio (SINR) was proposed in [11]. The pricing strategies for power control in multi-cell wireless data networks was analyzed in [12].

Another solution may be via properly designing the training sequences that are also used as the cell IDs. Good training sequences are expected to have several properties including constant amplitude, zero autocorrelation and orthogonality. For instance, the Zadoff-Chu (ZC) sequences used in 3GPPLTE (Long Term Evolution) have such nice properties [14]. However, the ZC sequences may not be suitable for multicell environments, because their orthogonality is destroyed in multipath channel environments, and this results in MCI. Also, when carrier frequency offset (CFO) appears, the induced MCI becomes serious. On the other hand, several multi-user interference (MUI-) free sequences have been proposed in [15]-[17]. Those sequences are shown to have interference-free property when there is no $\mathrm{CFO}$, and have negligible interference when CFO appears. The sequences in [17] attain a better PAPR (peak-to-average power ratio) performance than that in [15] and [16]. Although those sequences are interference free, they do not have the zero-autocorrelation property because the sequences are initially designed for MC-CDMA systems. From the discussion above, if we can find the sequences that enjoy constant amplitude, zero autocorrelation, and orthogonality in multipath channel environments, the handshaking procedure would be smooth in multi-cell networks. As a result, low-complexity cell ID detection, synchronization and CFO estimation algorithms designed for single-user or single-cell environments can be slightly modified and applied in multicell environments [18]-[26]. This motives us to design the sequences that have the mentioned nice properties.

In this paper, we propose training sequences for multi-cell environments. The proposed sequences enjoy the properties of constant amplitude, zero autocorrelation, and orthogonality in multipath channels both in time and frequency domains. The proposed sequences are inspired by the $\mathrm{ZC}$ sequences and the Gold code. We notice that Gold code has good orthogonality and the $\mathrm{ZC}$ sequences have the mentioned properties. By using similar procedures of generating the $\mathrm{ZC}$ sequences and the Gold code, the proposed sequences preserve the nice properties of these two sequences. More specifically, we select two ZC sequences and use similar shift-and-combine method for generating the proposed sequence. In addition, we theoretically prove that the proposed sequences can indeed achieve the mentioned properties. Moreover, we analyze the induced MCI when CFO appears, and find the MCI is negligible 


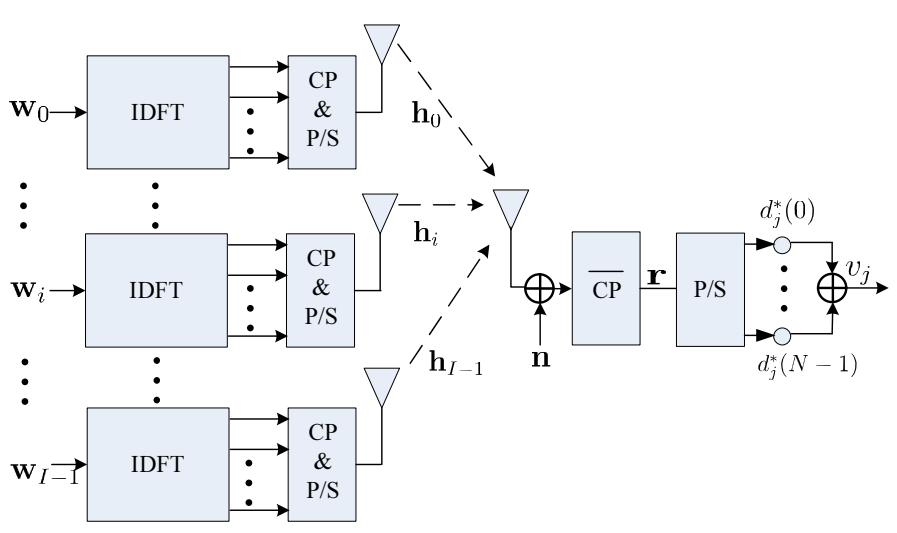

Fig. 1. The block diagram of a OFDM based multi-cell system.

in CFO environments. Furthermore, we take the single-cell CFO estimation algorithm in [19] as an example, and show how to modify it in multi-cell environments. As a result, the computational complexity for CFO and channel estimation can be greatly reduced thanks to the mitigation of MCI. Simulation results show that the analytical results are accurate, and the proposed sequences as well as the CFO estimation algorithms greatly outperform conventional schemes in multicell environments.

This paper is organized as follows: Sec. II describes the system model. In Sec. III, the generation of the proposed sequences and the analysis including autocorrelation and orthogonality are introduced. We analyze the MCI of the proposed sequences under multipath and $\mathrm{CFO}$ environments in Sec. IV, and propose the CFO estimation algorithm for multicell environments in Sec. V. Simulation results and conclusion are given in Secs. VI and VII, respectively.

Notations. All vectors are in lowercase boldface and matrices are in uppercase boldface. $(\cdot)^{T},(\cdot)^{*}$, and $(\cdot)^{H}$ denote the transpose, complex conjugate, and conjugate transpose of matrix. $((\cdot))_{N}$ and $|\cdot|$ represent the module of $N$ and absolute value, respectively. angle $(\cdot)$ denotes the phase. $\mathbf{I}_{L}$ is the $L \times L$ identity matrix. $\mathbf{P}_{(n)} \in \mathbb{R}^{N \times N}$ denotes a permutation matrix obtained by cyclically down shifting the row of an identity matrix $\mathbf{I}_{N}$ by $n$ elements. $\operatorname{diag}(\cdot)$ is a diagonal matrix which puts vector on the main diagonal.

\section{SySTEM MODEL AND PROBLEM FORMULATION}

A block diagram describing OFDM-based multi-cell cell search structure is illustrated in Fig. 1. Assume there are $I$ cells in the same frequency band and each cell is assigned a unique synchronization sequence $\mathbf{w}_{i}=\left(w_{i}[0] \cdots w_{i}[N-1]\right)^{T}$, $0 \leq i \leq I-1$. The synchronization sequence $\mathbf{w}_{i}$ is converted to $\mathbf{d}_{i}$ through inverse DFT (IDFT) operation, i.e., $\mathbf{d}_{i}=\mathbf{F}^{H} \mathbf{w}_{i}$, where $\mathbf{F}$ is the $N \times N$ DFT matrix with the $k$ th row and the $n$th column being $[\mathbf{F}]_{k n}=\frac{1}{\sqrt{N}} e^{-j \frac{2 \pi}{N} k n}$. Assume there is a user attempting to connect to one of these $I$ cells. For this user, the time-domain received signal after removing cyclic prefix $(\mathrm{CP})$ is represented as

$$
\mathbf{r}=\sum_{i=0}^{I-1} \boldsymbol{\Phi}_{i} \mathbf{H}_{i} \mathbf{d}_{i}+\mathbf{n},
$$

where $\boldsymbol{\Phi}_{i}=\operatorname{diag}\left(e^{j \frac{2 \pi}{N} N_{g} \epsilon_{i}} \ldots e^{j \frac{2 \pi}{N}\left(N+N_{g}-1\right) \epsilon_{i}}\right)$ is the CFO matrix with $N_{g}$ being the CP length and $\epsilon_{i}$ being the CFO between this user and the $i$ th cell. $\mathbf{n} \in \mathbb{C}^{N \times 1}$ is the noise vector and $\mathbf{H}_{i}$ is an $N \times N$ circulant matrix given by

$$
\mathbf{H}_{i}=\left(\begin{array}{lllll}
\mathbf{P}_{(0)} \overline{\mathbf{h}}_{i} & \mathbf{P}_{(1)} \overline{\mathbf{h}}_{i} & \cdots & \mathbf{P}_{(N-1)} \overline{\mathbf{h}}_{i}
\end{array}\right),
$$

where $\overline{\mathbf{h}}_{i}=\left[\begin{array}{ll}\mathbf{h}_{i}^{T} & \mathbf{0}_{1 \times N-L}\end{array}\right]^{T}$ and $\mathbf{h}_{i} \in \mathbb{C}^{L \times 1}$ are the channel of the $i$ th cell, with distribution $\mathcal{C N}(0,1)$. Since the circulant matrix $\mathbf{H}_{i}$ can be diagonalized using DFT and IDFT matrices, i.e., $\mathbf{H}_{i}=\mathbf{F}^{H} \boldsymbol{\Lambda}_{i} \mathbf{F}$, where $\boldsymbol{\Lambda}_{i}=\operatorname{diag}\left(\boldsymbol{\lambda}_{i}\right)$, and $\boldsymbol{\lambda}_{i}$ is the frequency response of the $i$ th channel expressed as $\boldsymbol{\lambda}_{i}=\mathbf{F} \overline{\mathbf{h}}_{i}$. We can rewrite (1) as

$$
\mathbf{r}=\sum_{i=0}^{I-1} \boldsymbol{\Phi}_{i} \mathbf{F}^{H} \boldsymbol{\Omega}_{i} \mathbf{F} \overline{\mathbf{h}}_{i}+\mathbf{n},
$$

where $\boldsymbol{\Omega}_{i}=\operatorname{diag}\left(\mathbf{w}_{i}\right)$. Our objective is to find a suitable cell for this user to connect, and complete the CFO estimation in the multi-cell environment. Thus it is important for this user to distinguish the signals between the targeted cell and the interfering cells. The targeted cell is chosen if it has the maximum matched value. Without loss of generality, assume the $j$ th cell is the targeted cell. The matched result can be expressed as

$$
\begin{aligned}
& \mathbf{d}_{j}^{H} \mathbf{r}=\mathbf{d}_{j}^{H}\left[\sum_{i=0}^{I-1} \boldsymbol{\Phi}_{i} \mathbf{F}^{H} \boldsymbol{\Omega}_{i} \mathbf{F} \overline{\mathbf{h}}_{i}+\mathbf{n}\right] \\
& =\{\underbrace{\mathbf{d}_{j}^{H} \boldsymbol{\Phi}_{j} \mathbf{F}^{H} \boldsymbol{\Omega}_{j} \mathbf{F} \overline{\mathbf{h}}_{j}}_{\text {desired signal }}+\sum_{i=0, i \neq j}^{I-1} \underbrace{\mathbf{d}_{j}^{H} \boldsymbol{\Phi}_{i} \mathbf{F}^{H} \boldsymbol{\Omega}_{i} \mathbf{F} \overline{\mathbf{h}}_{i}}_{\mathrm{MCI}_{j \leftarrow i}}+\mathbf{d}_{j}^{H} \mathbf{n}\} .
\end{aligned}
$$

The proposed sequence is to be used for cell detection under the environments with time and frequency offsets. In 3GPPLTE systems, the sequence for symbol timing, cell sector detection and CFO estimation is the primary synchronization sequence (PSS) [24],[25]. It is worth to point out that the PSS is designed for single cell environment. Thus, if it is used in multi-cell environments, the estimation performance degrades due to multi-cell interference (MCI). More specifically, from (3), the matched value is polluted by the MCI induced by multipath channels and CFO effects, and therefore it degrades the synchronization performance. One method to mitigate the MCI is to design the synchronization sequences that are robust to multipath channels and $\mathrm{CFO}$ effects. However, the PSS in LTE standard suffers from the MCI due to its weak orthogonality in time-domain. In order to mitigate the MCI as well as to preserve the utility of PSS, we propose a set of synchronization sequences which can simultaneously achieve good orthogonality and autocorrelation property. The proposed sequences can be used for cell detection under the multi-cell environments with frequency offsets.

\section{Proposed Synchronization Sequence}

The proposed sequence is motivated by the PSS applied in 3GPP-LTE systems and the idea of generating Gold sequence. 
We briefly introduce the PSS. In [1], the PSS $p_{u}$ is generated by the Zadoff-Chu (ZC) sequence of length 63, i.e.,

$$
p_{u}[k]=\left\{\begin{array}{l}
e^{-j \frac{\pi}{63} u k(k+1)}, \quad k=0,1, \ldots, 30 \\
e^{-j \frac{\pi}{63} u(k+1)(k+2)}, \quad k=31,32, \ldots, 61,
\end{array}\right.
$$

where $u=\{25,29,34\}$ is the root of the ZC sequence [14]. PSS has the properties of constant amplitude and zero autocorrelation (CAZAC) in both frequency domain and time domain. PSS is used for symbol timing, CFO estimation and cell sector detection [22]-[25]. In 3GPP-LTE systems, OFDMA scheme is adopted and one cell is camped in one specific frequency band. However, the cells in two-tier networks may occupy the same frequency band. As a result, using the PSS degrades the synchronization performance due to the MCI arisen from the lack of sequence orthogonality.

\section{A. Proposed sequence design}

To mitigate the MCI as well as to preserve the CAZAC property, we refer to the concept for generating the Gold sequence. Since ZC sequence has CAZAC property; while Gold sequences have good orthogonality, one intuition to generate a set of sequences that can preserve both properties is to combine the generation of these two kinds of sequences. More specifically, the proposed sequences are generated by combining two ZC sequences with different roots $u_{1}$ and $u_{2}$. The proposed sequences can be collected to form a matrix $\mathbf{W}=\left[\mathbf{w}_{0}, \mathbf{w}_{1}, \ldots, \mathbf{w}_{N-1}\right]$, where $\mathbf{w}_{i} \in \mathbb{C}^{N \times 1}$ is the synchronization sequence and is the combination of two ZC sequences $\mathbf{x}$ and $\mathbf{y}, 0 \leq i \leq N-1$. Each column in $\mathbf{W}$ is generated by the following formula

$$
\mathbf{w}_{i}=\mathbf{Y P}_{(i)} \mathbf{X}
$$

where $\mathbf{Y}=\operatorname{diag}(\mathbf{y})$ and $\mathbf{X}=\operatorname{diag}(\mathbf{x})$. For example, the proposed sequences for length $N=4$ are expressed as

$$
\mathbf{W}=\left(\begin{array}{llll}
y[0] x[0] & y[0] x[3] & y[0] x[2] & y[0] x[1] \\
y[1] x[1] & y[1] x[0] & y[1] x[3] & y[1] x[2] \\
y[2] x[2] & y[2] x[1] & y[2] x[0] & y[2] x[3] \\
y[3] x[3] & y[3] x[2] & y[3] x[1] & y[3] x[0]
\end{array}\right) .
$$

It is noted that the entry of the proposed sequences can be summarized as

$$
w_{i}[k]=e^{-j \frac{\pi}{N} \alpha_{i}[k]},
$$

where

$\alpha_{i}[k]=\left\{\begin{array}{l}u_{1}(k-i)^{2}+u_{2} k^{2}, \text { if } N \text { is even. } \\ u_{1}(k-i)(k-i+1)+u_{2} k(k+1), \text { if } N \text { is odd. }\end{array}\right.$

We show that the proposed sequence has good autocorrelation property and orthogonality, which can greatly simplify the computational complexity for CFO estimation and cell detection. Note that some of the proposed sequences are the cyclically shifted versions of the others, and this may lead to misjudgement because integer $\mathrm{CFO}$ may result in a cyclic shift of the proposed sequences in the frequency domain. Therefore, we analyze the cyclic property of the proposed sequences and propose a low-complexity method to identify the non-cyclically shifted sequences. The benefit of the method becomes more pronounced when $N$ grows large.

\section{B. Autocorrelation property of the proposed sequences}

In order to estimate the CFO and symbol timing, it is expected that the proposed sequences have zero autocorrelation in time domain. The following two lemmas and one theorem show that the proposed sequences have such property.

Lemma 1 The ZC sequence has the following property:

$$
x\left[((k-i))_{N}\right]=x[k-i] .
$$

Proof: Please see Appendix A.

Lemma 2 The proposed sequence defined in (4) has the following property:

$$
w_{i}\left[((k-n))_{N}\right]=w_{i}[k-n], \quad 1 \leq n \leq N-1 .
$$

Proof: Please see Appendix B.

Theorem 1 If the sum of the root indices $u_{1}+u_{2}$ and $N$ are coprime, the proposed sequence has zero autocorrelation in both time and frequency domains.

Proof: From (4), the frequency and time domain representations of the $i$ th column in $\mathbf{W}$ are expressed as $\mathbf{w}_{i}$ and $\mathbf{F}^{H} \mathbf{w}_{i}$, respectively. In time domain, zero autocorrelation means

$$
\left(\mathbf{F}^{H} \mathbf{w}_{i}\right)^{H}\left(\mathbf{F}^{H} \mathbf{P}_{(n)} \mathbf{w}_{i}\right)=0, \quad \text { for } 1 \leq n \leq N-1 .
$$

Since $\mathbf{F F}^{H}=\mathbf{I}_{N}$ the proof for time domain is similar to that for frequency domain. Therefore, we consider the analysis in frequency domain for simplicity. (7) can be rewritten as

$$
\sum_{k=0}^{N-1} w_{i}^{*}[k] w_{i}\left[((k-n))_{N}\right]=0, \text { for } 1 \leq n \leq N-1 .
$$

If $u_{1}+u_{2}$ and $N$ are coprime, from Lemma 2, the left hand side of (8) can be rewritten as

$$
\begin{aligned}
\sum_{k=0}^{N-1} w_{i}^{*}[k] w_{i}[k-n] & =\sum_{k=0}^{N-1} e^{j \frac{\pi}{N} \alpha_{i}[k]} e^{-j \frac{\pi}{N} \alpha_{i}[k-n]} \\
& =\sum_{k=0}^{N-1} e^{j \frac{\pi}{N}\left(\alpha_{i}[k]-\alpha_{i}[k-n]\right)}
\end{aligned}
$$

If $N$ is even, $\alpha_{i}[k]-\alpha_{i}[k-n]=-n^{2}\left(u_{1}+u_{2}\right)-2 n i u_{1}+$ $2 k n\left(u_{1}+u_{2}\right)$. Extracting the term irrelevant to $k$ yields

$$
\sum_{k=0}^{N-1} e^{j \frac{\pi}{N}\left(\alpha_{i}[k]-\alpha_{i}[k-n]\right)}=\beta_{1} \sum_{k=0}^{N-1} e^{j \frac{2 \pi}{N} k n\left(u_{1}+u_{2}\right)},
$$

where $\beta_{1}=e^{-j \frac{\pi}{N}\left[n^{2}\left(u_{1}+u_{2}\right)+2 n i u_{1}\right]}$. If $u_{1}+u_{2}$ and $N$ are coprime, $\sum_{k=0}^{N-1} e^{j \frac{2 \pi}{N} k n\left(u_{1}+u_{2}\right)}=0$, for $1 \leq n \leq N-1$. Similarly, it can also be shown that if $N$ is odd and $u_{1}+u_{2}$ and $N$ are coprime, the term in (9) is equal to zero. Therefore, the proposed sequence has zero autocorrelation if $u_{1}+u_{2}$ and $N$ are coprime.

\section{Orthogonality of the Proposed Sequences}

Like CDMA systems which use different orthogonal (or nearly orthogonal) spreading codes to distinguish different users, sequence orthogonality is an important property for cell detection in two-tier networks. We show the sequence orthogonality in the following theorem. 
Theorem 2 If the root index $u_{1}$ and $N$ are coprime, the proposed sequences are mutually orthogonal in both time and frequency domains.

Proof: To prove Thm. 2 is equivalent to proving $\mathbf{w}_{i}^{H} \mathbf{w}_{j}=$ 0 , and $\left(\mathbf{F}^{H} \mathbf{w}_{i}\right)^{H}\left(\mathbf{F}^{H} \mathbf{w}_{j}\right)^{H}=0$, where $i, j$ are the column indices of $\mathbf{W}, i \neq j$. Since $\mathbf{F F}^{H}=\mathbf{I}_{N}$, the analysis for time domain is similar to that for frequency domain. Therefore we analyze the orthogonality in frequency domain. We can rewrite $\mathbf{w}_{i}^{H} \mathbf{w}_{j}=0$ as

$$
\sum_{k=0}^{N-1} w_{i}^{*}[k] w_{j}[k]=0, \quad 0 \leq i, j \leq N-1, i \neq j .
$$

The left hand side of (11) can be expressed as

$$
\sum_{k=0}^{N-1} e^{j \frac{\pi}{N} \alpha_{i}[k]} e^{-j \frac{\pi}{N} \alpha_{j}[k]}=\sum_{k=0}^{N-1} e^{j \frac{\pi}{N}\left(\alpha_{i}[k]-\alpha_{j}[k]\right)} .
$$

If $N$ is even, $\alpha_{i}[k]-\alpha_{j}[k]=u_{1}\left(i^{2}-j^{2}\right)-2 k u_{1}(i-j)$. By extracting the term irrelevant to $k$, (12) can be rewritten as

$$
\beta_{2} \sum_{k=0}^{N-1} e^{-j \frac{2 \pi}{N} k u_{1}(i-j)}
$$

where $\beta_{2}=e^{j \frac{\pi}{N} u_{1}\left(i^{2}-j^{2}\right)}$. Since $0 \leq i-j \leq N-1$, if $u_{1}$ and $N$ are coprime, the term $\sum_{k=0}^{N-1} e^{-j \frac{2 \pi}{N} k u_{1}(i-j)}=0$. Similarly, if $N$ is odd, we can also prove that (11) holds if $u_{1}$ and $N$ are coprime, and this completes the proof.

\section{Cyclic shift analysis of the proposed sequences}

In previous sections, we have proven that the proposed sequences can simultaneously achieve zero autocorrelation and mutually orthogonal if $u_{1}+u_{2}$ and $u_{1}$ are prime to $N$. To estimate integer CFO [27],[28], the sequences should be designed so that none of them is the cyclically shifted version of others. For instance, if one sequences is a cyclic shifted version of another, the user cannot distinguish the two cells because he/she does not know whether the cyclic shift is caused by inter CFO or it is the sequence from another cell. Hence, we need to refine the proposed sequences so that they are not the cyclically shifted versions of the others. In this subsection, we analyze the cyclic shift property of the columns in $\mathbf{W}$, and propose a fast algorithm to determine which columns in $\mathbf{W}$ are non-cyclically shifted. Using this method, column by column search can be avoided and this can save a lot of computational complexity, especially when the size of $\mathbf{W}$ is large. The procedure is as follows: First, we show that every column in $\mathbf{W}$ have a symmetric point, that is, the column is cyclically symmetric at this symmetric point. Then we prove that if two columns in $\mathbf{W}$ have the same value at the symmetric points, these two columns are cyclically shifted to each other. Based on this cyclically symmetric property, we propose a low-complexity fast search method to find noncyclically shifted columns in $\mathbf{W}$.

Without loss of generality for practical implementations, let the sequence length $N=2^{s}, s \geq 2$. One setting that can meet the conditions in Thms. 1 and 2 is as the following proposition:
Proposition 1 The root indices $u_{1}$ and $u_{2}$ for generating the proposed sequences can be

$$
\left\{\begin{array}{l}
u_{1}=2 a+1, \quad a=0,1,2, \ldots, N-1 \\
u_{2}=2 b, \quad b=1,2, \ldots, N-1 .
\end{array}\right.
$$

Lemma 3 If $\mathbf{W}$ is generated by setting $u_{1}$ and $u_{2}$ be that in Proposition 1, there exists positive integers $\Delta$ and $g$ which satisfy $2\left[\left(u_{1}+u_{2}\right) \Delta-u_{1}\right]=g N$. Moreover, $\Delta$ is an odd number and can be expressed as $\Delta=2 p+1$, where $p$ is given by

$$
p=\frac{\frac{g N}{4}-b}{2 a+2 b+1} .
$$

Proof: $2\left[\left(u_{1}+u_{2}\right) \Delta-u_{1}\right]=g N$ can be reformulated as

$$
\left(u_{1}+u_{2}\right) \Delta-\frac{g N}{2}=u_{1} \text {. }
$$

Since $u_{1}+u_{2},-\frac{g N}{2}$ and $u_{1}$ are integers, (16) belongs to the Diophantine equation [29]. Moreover, $u_{1}+u_{2}$ is odd from Proposition 1; while $\frac{g N}{2}$ is even due to $N \geq 4$. Thus, the greatest common divisor (GCD) of $u_{1}+u_{2}$ and $-\frac{g N}{2}$ is 1 , and is able to divide $u_{1}$. Therefore, (16) exists an integer solution $\left(\Delta_{0}, g_{0}\right)$ and all the possible solutions can be obtained by

$$
\Delta=\Delta_{0}-\frac{g N}{2} t, \quad g=g_{0}-\left(u_{1}+u_{2}\right) t, \quad t \in \mathbb{Z} .
$$

From (16), it is clear that the solution $(\Delta, g)$ has the same parity since $\left(u_{1}+u_{2}\right)>u_{1}$. Therefore, $2\left[\left(u_{1}+u_{2}\right) \Delta-u_{1}\right]=$ $g N$ exists positive integer solution $(\Delta, g)$ if $t$ is properly determined. On the other hand, according to Proposition 1, $2\left[\left(u_{1}+u_{2}\right) \Delta-u_{1}\right]=g N$ can be rewritten as

$$
2[(2 a+2 b+1) \Delta-(2 a+1)]=g N .
$$

Since $(2 a+2 b+1)$ and $(2 a+1)$ are odd numbers and $N \geq 4$, $\Delta$ must be an odd number and can be expressed as $\Delta=2 p+1$. From (17), $p$ can be expressed as that in (15).

Theorem 3 For the ith column in $\mathbf{W}$, there always exists a symmetric point $k=i \Delta$, i.e.,

$w_{i}\left[((i \Delta+f))_{N}\right]=w_{i}\left[((i \Delta-f))_{N}\right]$, for $1 \leq f \leq N-1$.

Proof: From Lemma 2, $w_{i}\left[((i \Delta+f))_{N}\right]$ in (18) can be expressed as

$$
\begin{aligned}
& w_{i}[i \Delta+f]=e^{-j \frac{\pi}{N} \alpha_{i}[i \Delta+f]} \\
& =e^{-j \frac{\pi}{N}\left(u_{1}[(i \Delta-i)-f]^{2}+u_{2}(i \Delta-f)^{2}+4 f i\left[\left(u_{1}+u_{2}\right) \Delta-u_{1}\right]\right)} .
\end{aligned}
$$

According to Lemma 3 , the $\Delta$ in $4 f i\left[\left(u_{1}+u_{2}\right) \Delta-u_{1}\right]$ satisfies

$$
4 f i\left[\left(u_{1}+u_{2}\right) \Delta-u_{1}\right]=2 f i g N, \quad f, i \in \mathbb{Z}, g \in \mathbb{N} .
$$

Therefore, $w_{i}\left[((i \Delta+f))_{N}\right]$ can be expressed as

$$
\begin{aligned}
& w_{i}[i \Delta+f]=e^{-j \frac{\pi}{N}\left(u_{1}[(i \Delta-i)-f]^{2}+u_{2}(i \Delta-f)^{2}\right)} e^{-j \frac{2 \pi}{N} f i g N} \\
& =w_{i}[i \Delta-f] e^{-j 2 \pi f i g}=w_{i}[i \Delta-f]=w_{i}\left[((i \Delta-f))_{N}\right] .
\end{aligned}
$$

Note that the value of the symmetric point can be obtained by letting $f=0$, i.e.,

$$
w_{i}\left[((i \Delta))_{N}\right]=e^{-j \frac{\pi}{N} \alpha_{i}[i \Delta]},
$$


where $\alpha_{i}[i \Delta]=u_{1}(i \Delta-i)^{2}+u_{2}(i \Delta)^{2}$.

Lemma 4 The symmetric points of the columns of $\mathbf{W}$ generated by letting $b$ be odd have more unequal values than that generated by letting $b$ be even.

Proof: For the $i$ th and $j$ th columns in $\mathbf{W}$, the description that the values of the symmetric points are the same is equivalent to the following condition:

$$
w_{i}\left[((i \Delta))_{N}\right]=w_{j}\left[((j \Delta))_{N}\right] .
$$

From Lemma 2 and (21), we can rewrite (22) as

$$
e^{-j \frac{\pi}{N} \alpha_{i}[i \Delta]}=e^{-j \frac{\pi}{N} \alpha_{j}[j \Delta]} \equiv e^{-j \frac{\pi}{N}\left(\alpha_{i}[i \Delta]-\alpha_{j}[j \Delta]\right)}=1,
$$

where

$$
\begin{aligned}
\alpha_{i}[i \Delta]-\alpha_{j}[j \Delta] & =u_{1}\left[i^{2}(\Delta-1)^{2}-j^{2}(\Delta-1)^{2}\right] \\
& +u_{2}\left[i^{2} \Delta^{2}-j^{2} \Delta^{2}\right] \\
& =\left(i^{2}-j^{2}\right)\left[u_{1}(\Delta-1)^{2}+u_{2} \Delta^{2}\right] .
\end{aligned}
$$

From Proposition 1 and Lemma 3, $u_{1}(\Delta-1)^{2}+u_{2} \Delta^{2}$ in (24) can be expressed as

$$
\begin{aligned}
& u_{1}\left(\Delta^{2}-2 \Delta+1\right)+u_{2} \Delta^{2} \\
& =\Delta\left[\left(u_{1}+u_{2}\right) \Delta-u_{1}\right]-u_{1}(\Delta-1) \\
& =\Delta\left[\left(u_{1}+u_{2}\right) \Delta-u_{1}\right]-\left[\left(u_{1}+u_{2}\right) \Delta-u_{1}\right]+u_{2} \Delta \\
& =\frac{g N}{2}(2 p)+2 b(2 p+1)=2\left[p g \frac{N}{2}+b(2 p+1)\right] .
\end{aligned}
$$

Let $X(p, g, b)=p g(N / 2)+b(2 p+1)$, from (25), we can express (23) as

$$
e^{-j \frac{\pi}{N}\left\{\alpha_{i}[i \Delta]-\alpha_{j}[j \Delta]\right\}}=e^{-j \frac{2 \pi}{N}\left(i^{2}-j^{2}\right) X(p, g, b)}=1 .
$$

If $b$ is odd, $X(p, g, b)$ must be odd. To satisfy (26), we have

$$
i^{2}-j^{2}=c N, \quad c \in \mathbb{Z} .
$$

If $b$ is even, $p$ is even. The reason is that for $N \geq 4$, (15) can be rewritten as $\frac{g N}{4}-b=p(2 a+2 b+1)$. Since $(2 a+2 b+1)$ is odd and $\frac{g N}{4}$ is even, the integer value $p$ must also be even if $b$ is even. Therefore, $X(p, g, b)$ must be even and (26) can be rewritten as

$$
\begin{aligned}
e^{-j \frac{2 \pi}{N}\left(i^{2}-j^{2}\right) X(p, g, b)} & =e^{-j \pi p g\left(i^{2}-j^{2}\right)} e^{-j \frac{2 \pi}{N}\left(i^{2}-j^{2}\right) b(2 p+1)} \\
& =e^{-j \frac{2 \pi}{N}\left(i^{2}-j^{2}\right) b(2 p+1)}=1
\end{aligned}
$$

To satisfy (28), the following condition holds

$$
i^{2}-j^{2}=t \frac{N}{b}, \quad t \in \mathbb{Z} .
$$

Therefore, for the columns having the same value at their symmetric points, we can divide them into the following two cases according to if $b$ is even or odd:

$$
\begin{aligned}
& A=\left[(i, j) \mid i^{2}-j^{2}=c N, c \in \mathbb{Z}\right], \text { if } b \text { is odd } \\
& B=\left[(i, j) \mid i^{2}-j^{2}=t(N / b), t \in \mathbb{Z}\right], \text { if } b \text { is even. }
\end{aligned}
$$

Noted that $t(N / b) \in \mathbb{Z}$. Moreover, $b$ is even and therefore the possible indices in $B$ is larger than that in $A$, i.e., $A \subset B$. Therefore, letting $b$ be odd can obtain more unequal values at the symmetric points than letting $b$ be even.
Lemma 5 For the columns having the same value at their symmetric points, the indices $i$ and $j$ satisfy (30). In this case, if $i$ is even, $j$ is even; otherwise if $i$ is odd, $j$ is also odd.

Proof: When $b$ is odd, from (30) we have

$$
i^{2}-j^{2}=c N, \quad c \in \mathbb{Z} .
$$

Since $N$ is even, $c N$ is also even and therefore $i$ and $j$ must have the same parity.

When $b$ is even, from (30) we have

$$
i^{2}-j^{2}=t \frac{N}{b}, \quad t \in \mathbb{Z} .
$$

Since $b<N$ and $t(N / b) \in \mathbb{Z}, t(N / b)$ is even and $i$ and $j$ also have the same parity.

Theorem 4 For the $i$ and $j$ th columns having the same value at their symmetric points, i.e., $w_{i}\left[((i \Delta))_{N}\right]=w_{j}\left[((j \Delta))_{N}\right]$ in equation (22), the two columns are cyclically shifted.

Proof: To prove that columns $i$ and $j$ are cyclically shifted is to prove:

$$
w_{i}\left[((i \Delta+k))_{N}\right]=w_{j}\left[((j \Delta+k))_{N}\right], \quad 0 \leq k \leq N-1
$$

From Lemma 2 and equation (21), the left side of equation (31) can be rewritten as

$$
w_{i}\left[((i \Delta+k))_{N}\right]=w_{i}[i \Delta+k]=e^{-j \frac{\pi}{N} \alpha_{i}[i \Delta+k]},
$$

where $\alpha_{i}[i \Delta+k]$ is expressed as

$$
\begin{aligned}
& u_{1}(i \Delta+k-i)^{2}+u_{2}(i \Delta+k)^{2} \\
= & \underbrace{u_{1}(i \Delta-i)^{2}+u_{2}(i \Delta)^{2}}_{X_{1}(i, \Delta)}+\underbrace{2 k i\left[\left(u_{1}+u_{2}\right) \Delta-u_{1}\right]}_{X_{2}(i, \Delta, k)} \\
& +\underbrace{u_{1} k^{2}+u_{2} k^{2}}_{X_{3}(k)} .
\end{aligned}
$$

Therefore, from the condition that $w_{i}[i \Delta]=w_{j}[j \Delta], w_{i}[i \Delta+$ $k]$ can be expressed as

$$
\begin{aligned}
& e^{-j \frac{\pi}{N} X_{1}(i, \Delta)} e^{-j \frac{\pi}{N} X_{2}(i, \Delta, k)} e^{-j \frac{\pi}{N} X_{3}(k)} \\
& =w_{j}[j \Delta] e^{-j \frac{\pi}{N} X_{2}(i, \Delta, k)} e^{-j \frac{\pi}{N} X_{3}(k)} .
\end{aligned}
$$

From Lemma 3, we have

$$
e^{-j \frac{\pi}{N} X_{2}(i, \Delta, k)}=e^{-j \frac{\pi}{N} 2 k i\left[\left(u_{1}+u_{2}\right) \Delta-u_{1}\right]}=e^{-j \pi k i g} .
$$

From Lemma 5 , since $i$ and $j$ are positive integer and have same parity, it yields $e^{-j \pi k i g}=e^{-j \pi k j g}$ and $e^{-j \frac{\pi}{N} X_{2}(i, \Delta, k)}$ in (34) can be written as

$$
\begin{aligned}
e^{-j \frac{\pi}{N} X_{2}(i, \Delta, k)} & =e^{-j \frac{\pi}{N} 2 k i\left[\left(u_{1}+u_{2}\right) \Delta-u_{1}\right]} \\
& =e^{-j \frac{\pi}{N} 2 k j\left[\left(u_{1}+u_{2}\right) \Delta-u_{1}\right]}=e^{-j \frac{\pi}{N} X_{2}(j, \Delta, k) .}
\end{aligned}
$$

From (32), (34) and (36), $w_{i}\left[((i \Delta+k))_{N}\right]$ equals to

$$
\begin{aligned}
w_{i}[i \Delta+k] & =w_{j}[j \Delta] e^{-j \frac{\pi}{N} X_{2}(i, \Delta, k)} e^{-j \frac{\pi}{N} X_{3}(k)} \\
& =w_{j}[j \Delta] e^{-j \frac{\pi}{N} X_{2}(j, \Delta, k)} e^{-j \frac{\pi}{N} X_{3}(k)} \\
& =w_{j}[j \Delta+k]=w_{j}\left[((j \Delta+k))_{N}\right],
\end{aligned}
$$

which completes the proof.

From Lemma 4, Thms. 3 and 4, we conclude the following proposition: 
Proposition 2 If $u_{1}$ and $u_{2}$ are determined using Proposition 1 , the number of non-cyclically shifted columns in $\mathbf{W}$ by letting $b$ be even is more than that by letting $b$ be odd.

Fast algorithm to determine non-cyclically shifted columns. Based on the derived results in Lemma 4, Thms. 3 and 4, we propose a fast search algorithm to obtain the non-cyclically shifted columns in W. Instead of checking all elements of every column pair in $\mathbf{W}$, the proposed search algorithm only needs to check whether the symmetric points of the column pair are equal, i.e., whether $w_{i}\left[((i \Delta))_{N}\right]=$ $w_{j}\left[((j \Delta))_{N}\right]$. If the symmetric points of the column pair are equal, the two columns are cyclically shifted. Note that the proposed algorithm can reduce the computational complexity from $\mathcal{O}\left(C_{2}^{N} \times(N-1) \times N\right)$ to $\mathcal{O}\left(C_{2}^{N}\right)$. The proposed algorithm is summarized as follows:

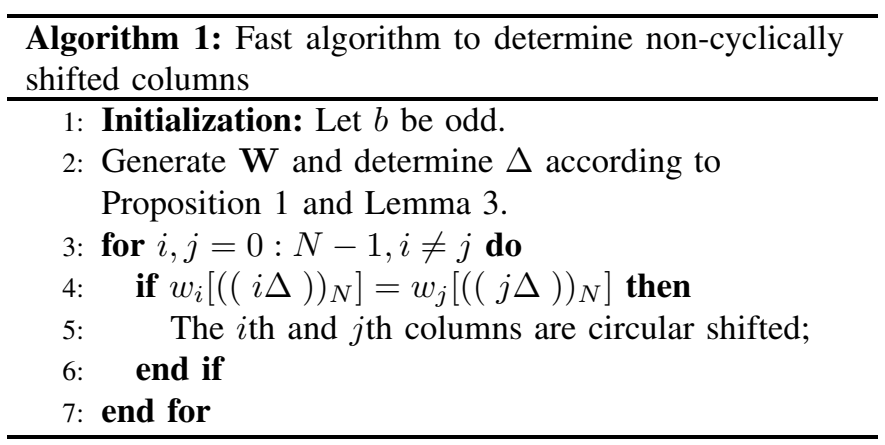

\section{Multi-CELl Interference AnALysis}

In multi-cell environments, the received signal contains the signals from different cells. Under multipath fading channels, the autocorrelation property and orthogonality discussed in Section III may be destroyed and this therefore induces multicell interference (MCI). MCI makes channel estimation and synchronization in multi-cell environments more difficult than that in single-cell environments. In this section, we analyze the MCI of the proposed sequences in multipath channels, and show that by properly grouping the proposed sequences, zero autocorrelation and mutually orthogonal property can still be preserved. It is worth to mention that this result stands in contrast to the PSS in the LTE standard, i.e., the autocorrelation and orthogonality of the PSS are no longer valid in multipath channels. Without loss of generality for practical implementations, the sequence length is set to be $N=2^{s}, s \geq 2$ throughout the MCI analysis.

\section{A. The MCI due to Multipath fading channels}

Since the channel is not known to the receiver during coarse synchronization stage, the MCI analysis is different from that in [15] and [16]. Here we show that the number of interfering cells using the proposed sequences is $L-1$ before channel compensation (usually called frequency-domain equalization in OFDM systems), and that is $2(L-1)$ after the channel compensation.
1) MCI analysis before channel compensation: As discussed in Section II, the MCI is induced by the multipath channels and the CFO effect. In the following analysis we first derive the $\mathrm{MCI}$ without $\mathrm{CFO}$, and then extend the results to $\mathrm{CFO}$ environment. According to (3), the MCI from the $i$ th cell to the user who links to the $j$ th cell can be expressed as

$$
\begin{aligned}
\mathbf{M C I}_{j \leftarrow i} & =\left(\mathbf{F}^{H} \mathbf{w}_{j}\right)^{H} \mathbf{F}^{H} \boldsymbol{\Omega}_{i} \mathbf{F} \overline{\mathbf{h}}_{i} \\
& =\mathbf{w}_{j}^{H} \boldsymbol{\Omega}_{i} \mathbf{F} \overline{\mathbf{h}}_{i}=\underbrace{\left[\mathbf{F}^{H} \boldsymbol{\Omega}_{i}^{*} \boldsymbol{\Omega}_{j} \mathbf{1}\right]^{H}\left(\begin{array}{c}
\mathbf{I}_{L} \\
\mathbf{0}
\end{array}\right)}_{\boldsymbol{\rho}_{j, i}} \mathbf{h}_{i},
\end{aligned}
$$

where 1 is an $N \times 1$ vector whose entries are all $1, \boldsymbol{\rho}_{j, i} \in$ $\mathbb{C}^{1 \times L}$. To have zero MCI for all nonzero $\mathbf{h}_{i}, \boldsymbol{\rho}_{j, i}$ must be a zero vector. Let $\boldsymbol{\mu}_{j, i}=\mathbf{F}^{H} \boldsymbol{\Omega}_{i}^{*} \boldsymbol{\Omega}_{j} \mathbf{1}$, and $\boldsymbol{\rho}_{j, i}$ is expressed as

$\boldsymbol{\rho}_{j, i}=\left(\mu_{j, i}^{*}(0), \ldots, \mu_{j, i}^{*}(L-1), \ldots, \mu_{j, i}^{*}(N-1)\right)\left(\begin{array}{l}\mathbf{I}_{L} \\ \mathbf{0}\end{array}\right)$.

It is clear that $\boldsymbol{\rho}_{j, i}=\mathbf{0}^{1 \times L}$ if

$\mu_{j, i}^{*}(n)=\frac{1}{\sqrt{N}} \sum_{k=0}^{N-1} w_{i}[k] w_{j}^{*}[k] e^{-j \frac{2 \pi}{N} k n}=0, \quad 0 \leq n \leq L-1$.

Theorem 5 Followed the assumptions in Sec.III-D that $N=$ $2^{s}$ and $u_{2}=2 b$. If the root $u_{1}$ for generating $\mathbf{W}$ is odd, the user who links to the targeted cell $j$ receives interference from only $L-1$ interfering cells with indices $i$ determined by the following equation:

$$
\left(\left(u_{1}(i-j)\right)\right)_{N}=n, \quad 1 \leq n \leq L-1 .
$$

Proof: From (5), $\mu_{j, i}^{*}$ in (39) is expressed as

$$
\begin{aligned}
\mu_{j, i}^{*}(n) & =\frac{1}{\sqrt{N}} \sum_{k=0}^{N-1} e^{-j \frac{\pi}{N} \alpha_{i}[k]} e^{j \frac{\pi}{N} \alpha_{j}[k]} e^{-j \frac{2 \pi}{N} k n} \\
& =\frac{1}{\sqrt{N}} \sum_{k=0}^{N-1} e^{j \frac{2 \pi}{N}\left(\frac{\alpha_{j}[k]-\alpha_{i}[k]}{2}-k n\right)} .
\end{aligned}
$$

Since $N$ is even, from (6), we have

$$
\begin{aligned}
\frac{\alpha_{j}[k]-\alpha_{i}[k]}{2}-k n & =\frac{u_{1}\left[(k-j)^{2}-(k-i)^{2}\right]}{2}-k n \\
& =k\left[u_{1}(i-j)-n\right]+\frac{u_{1}}{2}\left(j^{2}-i^{2}\right) .
\end{aligned}
$$

Therefore, $\mu_{j, i}^{*}(n)$ in (41) can be represented as

$$
\begin{aligned}
& \frac{1}{\sqrt{N}} e^{j \frac{\pi}{N} u_{1}\left(j^{2}-i^{2}\right)} \sum_{k=0}^{N-1} e^{j \frac{2 \pi}{N} k\left[u_{1}(i-j)-n\right]} \\
= & \left\{\begin{array}{cc}
\sqrt{N} e^{j \frac{\pi}{N} u_{1}\left(j^{2}-i^{2}\right)}, & \text { if } u_{1}(i-j)-n=g N, g \in \mathbb{Z} \\
0, & \text { otherwise. }
\end{array}\right.
\end{aligned}
$$

Without loss of generality, let $g=0$. The system has MCI if $\mu_{j, i}^{*}(n) \neq 0,1 \leq n \leq L-1$, which means that the column indices $(i, j)$ satisfy $u_{1}(i-j)-n=g N, g \in \mathbb{Z}$. This is equivalent to say $\left(\left(u_{1}(i-j)\right)\right)_{N}=n, 1 \leq n \leq L-1$. Moreover, the set consists of $(((i-j)))_{N}$ is equal to the set consists of $\left(\left(u_{1}(i-j)\right)\right)_{N}$, and they both have one-toone mapping if $u_{1}$ is odd. More specifically, given $u_{1}$ and the 
targeted cell index $j$, the $L-1$ indices of $i$ can be determined by varying the integer $n$ from 1 to $L-1$, and those indices $i$ are all different in the region $[0 N-1]$, i.e., there are $L-1$ interfering cells to the targeted cell.

2) MCI analysis after channel compensation: Similar to the derivation in [15], the MCI after channel compensation can be expressed as

$$
\mathrm{MCI}_{j \leftarrow i}=\mathbf{h}_{j}^{H} \underbrace{\mathbf{F}_{0}^{H} \boldsymbol{\Omega}_{j}^{*} \boldsymbol{\Omega}_{i} \mathbf{F}_{0}}_{\boldsymbol{\Gamma}_{j, i}} \mathbf{h}_{i}
$$

where

$$
\begin{gathered}
\boldsymbol{\Gamma}_{j, i}=\left(\begin{array}{ccc}
\gamma_{j, i}(0) & \ldots & \gamma_{j, i}(N-L+1) \\
\gamma_{j, i}(1) & \ldots & \gamma_{j, i}(N-L+2) \\
\vdots & \ddots & \vdots \\
\gamma_{j, i}(L-1) & \ldots & \gamma_{j, i}(0)
\end{array}\right)_{L \times L} \\
\gamma_{j, i}(n)=\frac{1}{\sqrt{N}} \sum_{k=0}^{N-1} w_{i}[k] w_{j}^{*}[k] e^{j \frac{2 \pi}{N} k n}
\end{gathered}
$$

and $\mathbf{F}_{0}=\mathbf{F}\left(\begin{array}{c}\mathbf{I}_{L} \\ \mathbf{0}\end{array}\right)_{N \times L}$. Therefore, having zero $\mathbf{M C I}_{j \leftarrow i}$ is equivalent to let $\boldsymbol{\Gamma}_{j, i}=\mathbf{0}$, i.e.,

$$
\begin{cases}\gamma_{j, i}(n)=0, & 0 \leq n \leq L-1 \\ \gamma_{j, i}(N-n)=0, & 1 \leq n \leq L-1,\end{cases}
$$

Theorem 6 If the root $u_{1}$ for generating $\mathbf{W}$ is odd, the number of interfering cells is $2(L-1)$ after channel compensation. For the targeted cell $j$, the index of interfering cell $i$ can be determined by

$$
\begin{cases}\left(\left(u_{1}(i-j)\right)\right)_{N}=n, & 1 \leq n \leq L-1 . \\ \left(\left(u_{1}(i-j)\right)\right)_{N}=N-n, & 1 \leq n \leq L-1 .\end{cases}
$$
omitted.

Proof: The proof is similar to that of Thm. 5 and is

Although the interfering cells of a targeted cell can be obtained through Thms. 5 and 6 in multipath channels, determining the sequences that are mutually orthogonal is crucial because this property not only can mitigate the MCI effect, but also can simplify the design complexity of CFO estimation. In Thm. 2, we have proved the mutually orthogonal property of the proposed sequences in flat fading channels. In the following corollary we show that the proposed sequences can preserve the mutually orthogonal property in multipath channels if the sequence indices are appropriately selected.

Corollary 1 Let the root $u_{1}$ for generating $\mathbf{W}$ be odd and the sequence length $N=2^{s}, s \geq 2$. Let the parameter $G$ be $G=2^{s^{\prime}} \geq L, s^{\prime} \in \mathbb{N}$. The number of mutually orthogonal cells is $N / G$. More specifically, if cell $j$ is selected, the indices of the other mutually orthogonal cells are

$$
i=((j+o G))_{N}, o \in \mathbb{Z} .
$$

Proof: From Thm. 5, there are $L-1$ interfering cells to the cell $j$ and the corresponding indices can be obtained through

$$
(((j-i)))_{N}=n, \quad 1 \leq n \leq L-1 .
$$

For $u_{1}=1$, the cell $j$ is interfered by the cells with indices $i=((j+\tau))_{N}, 1 \leq \tau \leq L-1$, i.e., the neighboring
$L-1$ cell indices. Therefore, if we select the cell indices $i^{\prime}$ that are uniformly separated by a period $G \geq L$, i.e., $i^{\prime}=$ $j+o G, o \in \mathbb{Z}$, these cells would not interfere with each other. In other words, they are mutually orthogonal.

For odd number $u_{1} \neq 1$, the interfering cell indices to the cell $j$ can be obtained through $i=j+(n+g N) / u_{1}, 1 \leq$ $n \leq L-1, g \in \mathbb{Z}$. Since

$n+g N=n+g 2^{s}=n+g\left(2^{s-s^{\prime}}\right) G \neq u_{1} o G$, for $1 \leq n \leq L-1$,

the selected indices $i^{\prime}$ would not become an interferer. More specifically, $i^{\prime}=j+o G \neq j+(n+g N)=i$, for $1 \leq n \leq$ $L-1$. Therefore, they are still mutually orthogonal.

\section{B. The MCI of the proposed sequence due to CFO effect}

In this subsection, we consider the MCI due to carrier frequency offset (CFO). In two-tier networks, the CFO effect makes the user difficult to synchronize. More specifically, the phase difference from different transmit branches in the time domain would complicate the CFO estimation and therefore degrade the synchronization performance. The MCI induced by the $\mathrm{CFO}$ can be divided into two terms, i.e., the dominant MCI and the residual MCI. We show that if the proposed sequences are used, the dominant MCI can be completely eliminated and the residual MCI is mitigated into a negligible level. Therefore, we can still apply the CFO estimation algorithm designed for single-user or single-cell environments to the multi-cell environments. As a result, not only the estimation performance can be improved but also the computational complexity can be greatly reduced. From (3), the $\mathrm{MCI}_{j \leftarrow i}$ with $\mathrm{CFO}$ is expressed as

$$
\mathbf{M C I}_{j \leftarrow i}=\mathbf{d}_{j}^{H} \boldsymbol{\Phi}_{i} \mathbf{F}^{H} \boldsymbol{\Omega}_{i} \mathbf{F} \overline{\mathbf{h}}_{i}=\mathbf{d}_{j}^{H} \boldsymbol{\Phi}_{i} \mathbf{F}^{H} \boldsymbol{\Omega}_{i} \boldsymbol{\lambda}_{i} .
$$

(48) can be further expanded to (49), depicted at the top of the next page. According to [15], $X(k, m)$ in (49) can be expressed as

$$
\begin{aligned}
X(k, m) & =\alpha_{i} \lambda_{i}[k] w_{i}[k] \\
& +\beta_{i} \sum_{u=m, m \neq k}^{N-1} \lambda_{i}[m] w_{i}[m] \frac{e^{-j \pi \frac{m-k}{N}}}{N \sin \frac{\pi\left(m-k+\epsilon_{i}\right)}{N}},
\end{aligned}
$$

where

$$
\alpha_{i}=\frac{\sin \pi \epsilon_{i}}{N \sin \frac{\pi \epsilon_{i}}{N}} e^{j \pi \epsilon_{i} \frac{N-1}{N}} \text { and } \beta_{i}=\sin \left(\pi \epsilon_{i}\right) e^{j \pi \epsilon_{i} \frac{N-1}{N}} .
$$

Therefore, $\mathrm{MCI}_{j \leftarrow i}$ can be divided into $\mathrm{MCI}_{j \leftarrow i \text {,domi }}$ and $\mathrm{MCI}_{j \leftarrow i \text {,resi }}$, shown at the bottom of the next page. Note that when there is no CFO between the $i$ th cell and the user, $\alpha_{i}=1$ and $\beta_{i}=0$, and the $\mathrm{MCI}_{j \leftarrow i}$ reduces to $\mathrm{MCI}_{j \leftarrow i \text {,domi }}$. This result gives us an intuition that the overall MCI is determined by $\mathrm{MCI}_{j \leftarrow i \text {,domi }}$ when the CFO is small. Therefore, the MCI information of the targeted cell with $\mathrm{CFO}$ is similar to that without CFO. Moreover, if we can eliminate the dominant MCI, the overall MCI can be sufficiently reduced. Similar to (38), $\mathrm{MCI}_{j \leftarrow i \text {, domi }}$ can be expressed as $\mathrm{MCI}_{j \leftarrow i \text {,domi }}=$ $\alpha_{i} e^{j \frac{2 \pi}{N} N_{g} \epsilon_{i}} \mathbf{w}_{j}^{H} \boldsymbol{\Omega}_{i} \mathbf{F} \overline{\mathbf{h}}_{i}$. From Thm. 5, $\mathbf{M C I}_{j \leftarrow i \text {,domi can be }}$ completed eliminated if the proposed sequences are appropriately selected. This result motivates the proposed multi-cell $\mathrm{CFO}$ estimation algorithm in the following section. 


$$
\begin{aligned}
\operatorname{MCI}_{j \leftarrow i} & =\sum_{n=0}^{N-1}\left\{\left[\frac{1}{\sqrt{N}} \sum_{k=0}^{N-1} w_{j}[k] e^{j \frac{2 \pi}{N} n k}\right]^{*}\left[\frac{1}{\sqrt{N}} \sum_{m=0}^{N-1} w_{i}[m] \lambda_{i}[m] e^{j \frac{2 \pi}{N} n m} e^{j \frac{2 \pi}{N}\left(n+N_{g}\right) \epsilon_{i}}\right]\right\} \\
& =e^{j \frac{2 \pi}{N} N_{g} \epsilon_{i}} \sum_{n=0}^{N-1}\left\{\frac{1}{\sqrt{N}} \sum_{k=0}^{N-1}\left[w_{j}^{*}[k] e^{-j \frac{2 \pi}{N} k n} \frac{1}{\sqrt{N}} \sum_{m=0}^{N-1} w_{i}[m] \lambda_{i}[m] e^{j \frac{2 \pi}{N} n m} e^{j \frac{2 \pi}{N} n \epsilon_{i}}\right]\right\} \\
& =e^{j \frac{2 \pi}{N} N_{g} \epsilon_{i}} \sum_{k=0}^{N-1} w_{j}^{*}[k] \underbrace{\left\{\frac{1}{\sqrt{N}} \sum_{n=0}^{N-1}\left[\frac{1}{\sqrt{N}} e^{-j \frac{2 \pi}{N} n k} \sum_{m=0}^{N-1} w_{i}[u] \lambda_{i}[m] e^{j \frac{2 \pi}{N} n m} e^{j \frac{2 \pi}{N} n \epsilon_{i}}\right]\right\}}_{X(k, m)} .
\end{aligned}
$$

\section{CARrier Frequency OfFset Estimation}

Based on the derived MCI results for the proposed sequences, we propose a multi-cell CFO estimation algorithm in this section. We first introduce the CFO estimation algorithm applied to this paper in single-cell environments, and then extend the algorithm to multi-cell environments. In multi-cell environments, the CFO estimation performance degrades due to MCI. By using the proposed sequences and algorithm, the MCI can be effectively mitigated and the CFO estimation in multi-cell environments is same as that in single-cell environments, which can greatly reduce the computational complexity. Moreover, we also compare the performance of CFO estimation between the proposed sequences and the PSS.

\section{A. Single-cell CFO estimation}

Let us take the 3GPP-LTE synchronization procedure as an instance. In this case, the CFO can be estimated by applying the Schmidl scheme [19], and the acquisition is achieved in two separate steps through the use of a twosymbol synchronization sequences. The Schmidl algorithm is explained as follows: Two consecutive received signals are expressed as

$$
\mathbf{r}^{(1)}=\boldsymbol{\Phi}^{(1)} \mathbf{F}^{H} \boldsymbol{\Lambda} \mathbf{w}+\mathbf{n}^{(1)} \text { and } \mathbf{r}^{(2)}=\boldsymbol{\Phi}^{(2)} \mathbf{F}^{H} \boldsymbol{\Lambda} \mathbf{w}+\mathbf{n}^{(2)},
$$

where $\boldsymbol{\Phi}^{(1)}$ and $\boldsymbol{\Phi}^{(2)}$ are diagonal CFO matrices whose elements are respectively expressed as $\left[\boldsymbol{\Phi}^{(1)}\right]_{n n}=e^{j \frac{2 \pi}{N}\left(n+N_{g}\right) \epsilon}$ and $\left[\boldsymbol{\Phi}^{(2)}\right]_{n n}=e^{j \frac{2 \pi}{N}\left(n+N+2 N_{g}\right) \epsilon}, 0 \leq n \leq N-1$. Then the systems perform the inner product of $\mathbf{r}^{(1)}$ and $\mathbf{r}^{(2)}$ to obtain q, i.e., $q$ is obtained through

$$
\left[\mathbf{r}^{(1)}\right]^{H} \mathbf{r}^{(2)}=\left[\boldsymbol{\Phi}^{(1)} \mathbf{F}^{H} \boldsymbol{\Lambda} \mathbf{w}+\mathbf{e}^{(1)}\right]^{H}\left[\boldsymbol{\Phi}^{(2)} \mathbf{F}^{H} \boldsymbol{\Lambda} \mathbf{w}+\mathbf{e}^{(2)}\right]
$$

Under high SNR assumption, $\mathbf{e}^{(1)}$ and $\mathbf{e}^{(2)}$ can be ignored. Thus, (50) can be reduced to

$$
\begin{aligned}
q & =\left[\mathbf{\Phi}^{(1)} \mathbf{F}^{H} \boldsymbol{\Lambda} \mathbf{w}\right]^{H}\left[\boldsymbol{\Phi}^{(2)} \mathbf{F}^{H} \boldsymbol{\Lambda} \mathbf{w}\right] \\
& =\mathbf{w}^{H} \boldsymbol{\Lambda}^{H} \mathbf{F}\left[\boldsymbol{\Phi}^{(1)}\right]^{H} \boldsymbol{\Phi}^{(2)} \mathbf{F}^{H} \boldsymbol{\Lambda} \mathbf{w} .
\end{aligned}
$$

It is clear that $\boldsymbol{\Phi}^{(2)}=e^{j 2 \pi \epsilon\left(1+\frac{N_{g}}{N}\right)} \boldsymbol{\Phi}^{(1)}$ and (51) can be further rewritten as

$$
\begin{aligned}
q & =e^{j 2 \pi \epsilon\left(1+\frac{N_{g}}{N}\right)} \mathbf{w}^{H} \boldsymbol{\Lambda}^{H} \mathbf{F}\left[\mathbf{\Phi}^{(1)}\right]^{H} \boldsymbol{\Phi}^{(1)} \mathbf{F}^{H} \boldsymbol{\Lambda} \mathbf{w} \\
& =e^{j 2 \pi \epsilon\left(1+\frac{N_{g}}{N}\right)}\|\boldsymbol{\Lambda} \mathbf{w}\|^{2} .
\end{aligned}
$$

Therefore, the estimated CFO $\widehat{\epsilon}$ can be obtained given by

$$
\widehat{\epsilon}=\frac{1}{2 \pi\left(1+\frac{N_{g}}{N}\right)} \operatorname{angle}(q) .
$$

\section{B. Multi-cell CFO estimation}

In multi-cell environments, the MCI would affect the accuracy of CFO estimation. In this case, the Schmidl scheme may not be directly applied to estimate CFO. To overcome this issue, we propose an CFO estimation algorithm based on the proposed sequences for multi-cell environments. It is noted that this algorithm is modified from the Schmidl scheme. From (1), two consecutively received signals are expressed as

$$
\begin{aligned}
& \mathbf{r}^{(1)}=\sum_{i=0}^{I-1} \underbrace{\boldsymbol{\Phi}_{i}^{(1)} \mathbf{F}^{H} \boldsymbol{\Lambda}_{i} \mathbf{w}_{i}}_{\mathbf{s}_{i}^{(1)}}+\mathbf{n}^{(1)} \\
& \mathbf{r}^{(2)}=\sum_{i=0}^{I-1} \underbrace{\boldsymbol{\Phi}_{i}^{(2)} \mathbf{F}^{H} \boldsymbol{\Lambda}_{i} \mathbf{w}_{i}}_{\mathbf{s}_{i}^{(2)}}+\mathbf{n}^{(2)},
\end{aligned}
$$

where $\boldsymbol{\Phi}_{i}^{(1)}$ and $\boldsymbol{\Phi}_{i}^{(2)}$ are diagonal CFO matrices whose elements are respectively represented as $\left[\boldsymbol{\Phi}_{i}^{(1)}\right]_{n n}=$ $e^{j \frac{2 \pi}{N}\left(n+N_{g}\right) \epsilon_{i}}$ and $\left[\boldsymbol{\Phi}_{i}^{(2)}\right]_{n n}=e^{j \frac{2 \pi}{N}\left(n+N+2 N_{g}\right) \epsilon_{i}}, 0 \leq n \leq$ $N-1$. $\mathbf{n}^{(1)}$ and $\mathbf{n}^{(2)}$ are thermal noises and can be ignored at high SNR.

To explicitly explain why the Schmidl scheme may not work in multi-cell environments, we give an example which consists of three cells in the same frequency band. Moreover, we assume the user already determines which cell to link before the CFO estimation; this assumption is reasonable because cell ID is in general determined before CFO estimation. Also, we

$$
\mathbf{M C I}_{j \leftarrow i}=\underbrace{\alpha_{i} e^{j \frac{2 \pi}{N} N_{g} \epsilon_{i}} \sum_{k=0}^{N-1} \lambda_{i}[k] w_{i}[k] w_{j}^{*}[k]}_{\mathbf{M C I}_{j \leftarrow i \text {,omi }}}+\underbrace{\beta_{i} e^{j \frac{2 \pi}{N} N_{g} \epsilon_{i}} \sum_{k=0}^{N-1} \sum_{m=0, m \neq k}^{N-1} \lambda_{i}[m] w_{i}[m] w_{j}^{*}[k] \frac{e^{-j \pi \frac{m-k}{N}}}{N \sin \frac{\pi\left(m-k+\epsilon_{i}\right)}{N}}}_{\mathbf{M C I}_{j \leftarrow i \text {,resi }}} .
$$


consider the system is at high SNR. Therefore, the received signals can be expressed as

$$
\begin{aligned}
& \mathbf{r}^{(1)}=\mathbf{s}_{1}^{(1)}+\mathbf{s}_{2}^{(1)}+\mathbf{s}_{3}^{(1)} \\
& \mathbf{r}^{(2)}=\mathbf{s}_{1}^{(2)}+\mathbf{s}_{2}^{(2)}+\mathbf{s}_{3}^{(2)}
\end{aligned}
$$

If the Schmidl algorithm in (50) is applied to estimate the $\mathrm{CFO}$ of cell $1, q$ is given by

$$
\begin{aligned}
q & =\left[\mathbf{r}^{(1)}\right]^{H} \mathbf{r}^{(2)}=\left[\mathbf{s}_{1}^{(1)}+\mathbf{s}_{2}^{(1)}+\mathbf{s}_{3}^{(1)}\right]^{H}\left[\mathbf{s}_{1}^{(2)}+\mathbf{s}_{2}^{(2)}+\mathbf{s}_{3}^{(2)}\right] \\
& =\left[\mathbf{s}_{1}^{(1)}\right]^{H} \mathbf{s}_{1}^{(2)}+\left[\mathbf{s}_{2}^{(1)}\right]^{H} \mathbf{s}_{2}^{(2)}+\left[\mathbf{s}_{3}^{(1)}\right]^{H} \mathbf{s}_{3}^{(2)} \\
& +\sum_{j=1}^{3} \sum_{i=1, i \neq j}^{3}\left[\mathbf{s}_{j}^{(1)}\right]^{H} \mathbf{s}_{i}^{(2)}
\end{aligned}
$$

where the term $\left[\mathbf{s}_{j}^{(1)}\right]^{H} \mathbf{s}_{i}^{(2)}$ represents the MCI. With the proposed sequences, from Thm. $5,\left[\mathbf{s}_{j}^{(1)}\right]^{H} \mathbf{s}_{i}^{(2)}=0$ by appropriately grouping the sequences; while this term is not zero for the PSS. Thus, our proposed sequences have better CFO estimation accuracy than the PSS when the Schmidl algorithm is adopted. However, even using the proposed sequences in the Schmidl scheme, there still exists the interference terms $\left[\mathbf{s}_{2}^{(1)}\right]^{H} \mathbf{s}_{2}^{(2)}$ and $\left[\mathbf{s}_{3}^{(1)}\right]^{H} \mathbf{s}_{3}^{(2)}$. Therefore, we propose a method to mitigate the interferences to improve the CFO estimation accuracy. More specifically, $\mathbf{r}^{(1)}$ and $\mathbf{r}^{(2)}$ are both multiplied by the Hermitian of the time domain synchronization sequence for the targeted cell before performing inner product. Below we explain how to mitigate the MCI by applying the proposed method for CFO estimation:

The value $q$ using the proposed method is expressed as

$$
q=\left[\mathbf{d}_{j}^{H} \mathbf{r}^{(1)}\right]^{H}\left[\mathbf{d}_{j}^{H} \mathbf{r}^{(2)}\right]
$$

From (1), (54) can be expressed to (55), shown at the bottom of the next page, and it can be manipulated as

$$
\begin{aligned}
& {\left[\mathbf{d}_{j}^{H} \boldsymbol{\Phi}_{j}^{(1)} \mathbf{F}^{H} \boldsymbol{\Lambda}_{j} \mathbf{w}_{j}+\sum_{i=0, i \neq j}^{I-1} \mathrm{MCI}_{j \leftarrow i, \text { domi }}^{(1)}+\mathrm{MCI}_{j \leftarrow i, \text { resi }}^{(1)}\right]^{H}} \\
& \cdot\left[\mathbf{d}_{j}^{H} \boldsymbol{\Phi}_{j}^{(2)} \mathbf{F}^{H} \boldsymbol{\Lambda}_{j} \mathbf{w}_{j}+\sum_{i=0, i \neq j}^{I-1} \mathrm{MCI}_{j \leftarrow i, \text { domi }}^{(2)}+\mathrm{MCI}_{j \leftarrow i, \text { resi }}^{(2)}\right] .
\end{aligned}
$$

From the discussion in IV, with the proposed sequences, $\mathrm{MCI}_{j \leftarrow i \text {, resi }}$ is sufficiently small to be ignored when CFO is small. Moreover, $\mathrm{MCI}_{j \leftarrow i \text {, domi }}$ is zero by appropriately grouping the proposed sequences. Therefore, (56) can be approximated to

$$
\begin{aligned}
q & \approx\left[\mathbf{d}_{j}^{H} \boldsymbol{\Phi}_{j}^{(1)} \mathbf{F}^{H} \boldsymbol{\Lambda}_{j} \mathbf{w}_{j}\right]^{H}\left[\mathbf{d}_{j}^{H} \boldsymbol{\Phi}_{j}^{(2)} \mathbf{F}^{H} \boldsymbol{\Lambda}_{j} \mathbf{w}_{j}\right] \\
& =e^{j 2 \pi \epsilon_{j}\left(1+\frac{N_{g}}{N}\right)}\left\|\mathbf{d}_{j}^{H} \boldsymbol{\Phi}_{j}^{(1)} \mathbf{F}^{H} \boldsymbol{\Lambda}_{j} \mathbf{w}_{j}\right\|^{2} .
\end{aligned}
$$

Therefore, the estimated CFO $\widehat{\epsilon}_{j}$ of the targeted cell can be obtained by

$$
\widehat{\epsilon}_{j}=\frac{1}{2 \pi\left(1+\frac{N_{g}}{N}\right)} \operatorname{angle}(q) \text {. }
$$

It is worth to emphasize that the proposed CFO estimation is modified from the Schmidl algorithm, which is designed for single cell environment. The proposed CFO estimation by using the designed sequences for multi-cell environments in concluded in Algorithm 2.

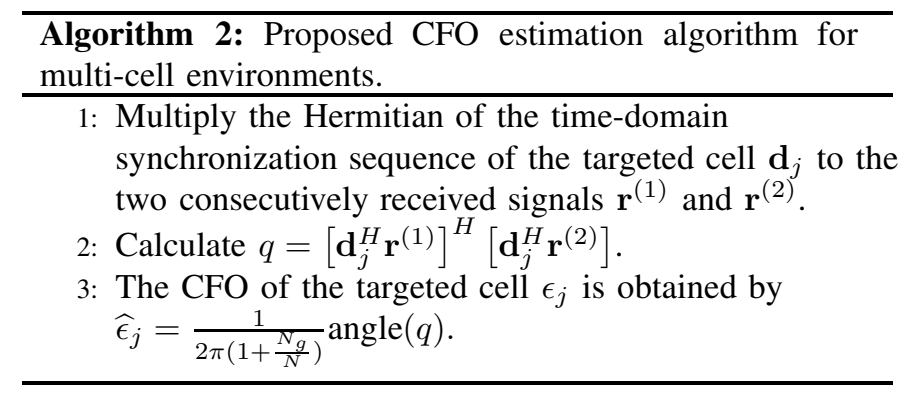

\section{Simulation RESUlts}

In this section, simulation results are provided to show the orthogonality and the MCI characteristics of the proposed sequences. Moreover, we compare the accuracy of the CFO estimation between the PSS [1] and the proposed sequences in single-cell and multi-cell environments. The sequence length $N$ is 64 and the roots for the proposed sequences are $u_{1}=3$ and $u_{2}=2$.

Example 1. Interfering cells of the proposed sequences: This example investigates the number of interfering cells in Thms 5 and 6 . Let the sequence length be $N=32$ and the multipath length be $L=4$. The MCI results before and after channel compensation are shown in Figs. 2(a) and 2(b), respectively. The black squares in the figures indicate that if the user links to the targeted cell $j$, he/she receives the interference from the interfering cells with index $i$. We observe that the number of interfering cells before channel compensation is $L-1$, which is consistent with the discussion in Section IV. On the other hand, the number of interfering cells is $2(L-1)$ after channel compensation, which also matches the analytical results.

Example 2. MCI of the proposed sequences with CFO: The MCI to the targeted cell for multipath length $L=4$ is illustrated in Fig. 3. The simulation result is obtained by setting the distribution of the CFO be either uniform or Gaussian, which may represent the worst and the realistic CFO scenarios, respectively. More specifically, we let the CFO be uniformly distributed between -0.1 and 0.1 (subcarrier spacing), or normally distributed with outage probability $\operatorname{Pr}\{|\mathrm{CFO}|>0.1\}=10^{-5}$. The MCI is normalized by $\left.\left|\sum_{k=0}^{N-1} \lambda_{j}[k]\right| w_{i}[k]\right|^{2} \mid$ and averaged for different channel realizations. Except for the dominant interfering cells, the gap between the desired signal and the most serious interfering cell are around $13 \mathrm{~dB}$ for uniform $\mathrm{CFO}$ and $18 \mathrm{~dB}$ for Gaussian $\mathrm{CFO}$; in general the gap are around $18 \mathrm{~dB}$ for uniform $\mathrm{CFO}$ and $23 \mathrm{~dB}$ for Gaussian CFO. By carefully excluding the most serious interfering cells, the induced interference is small and ignorable. Therefore using the proposed sequences, the algorithms designed for single-cell environments can be used in multi-cell environments even if $\mathrm{CFO}$ exists. 


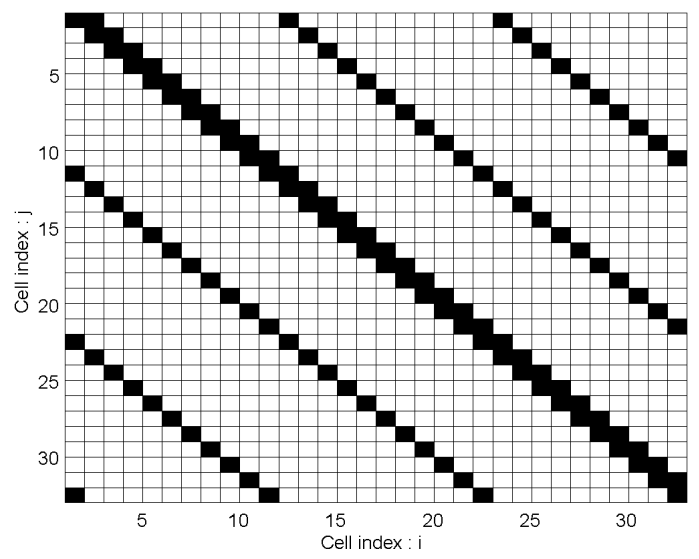

(a) Before channel equalization.

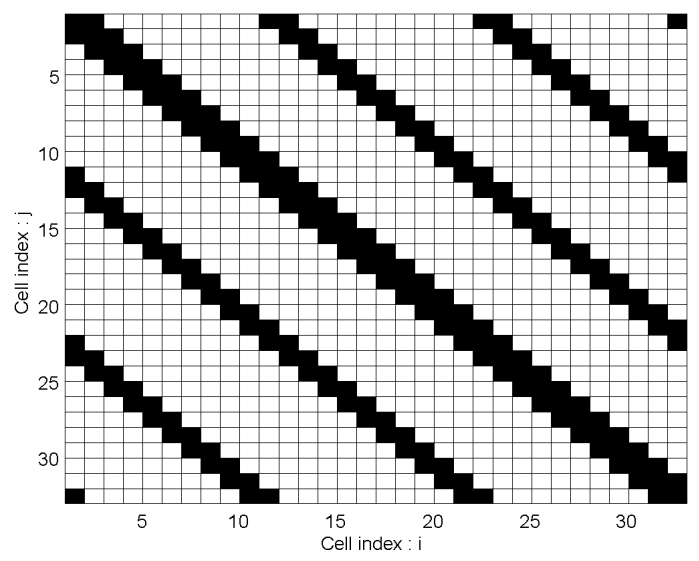

(b) After channel equalization.

Fig. 2. The MCI of the proposed sequences with multipath length $L=4$.

Example 3. CFO estimation in single-cell environments: This example shows the accuracy of CFO estimation for the proposed sequences in single-cell environments. Let the length of CP $N_{g}$ be 16, and the CFO distribution be either uniform or Gaussian like the settings in Example 2. To ensure the correctness of the simulation, first we let the channel be AWGN, where we can find a Cramer-Rao bound for the estimation error [19], which is

$$
\operatorname{var}\left[\frac{\widehat{\epsilon}}{2 \pi\left(1+\frac{N_{g}}{N}\right)}\right] \geq \frac{1}{\left[2 \pi\left(1+\frac{N_{g}}{N}\right)\right]^{2} \cdot N \cdot S N R} .
$$

The estimation error of the primary sequence and the proposed sequence for AWGN channel is shown in Fig. 4. We see that when SNR is sufficiently high, both the PSS and the proposed sequences can achieve the Cramer-Rao bound in

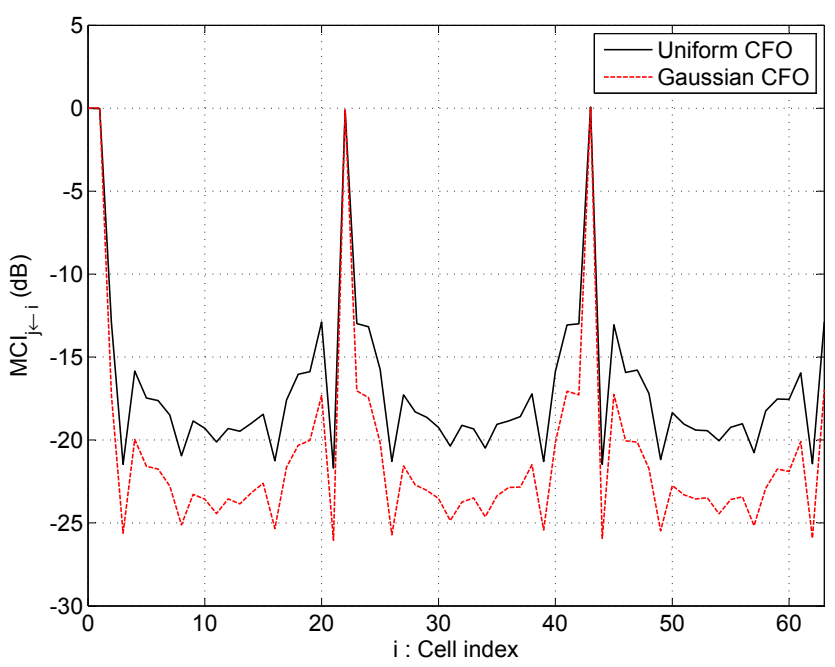

Fig. 3. The MCI of the proposed sequence with different CFO distributions.

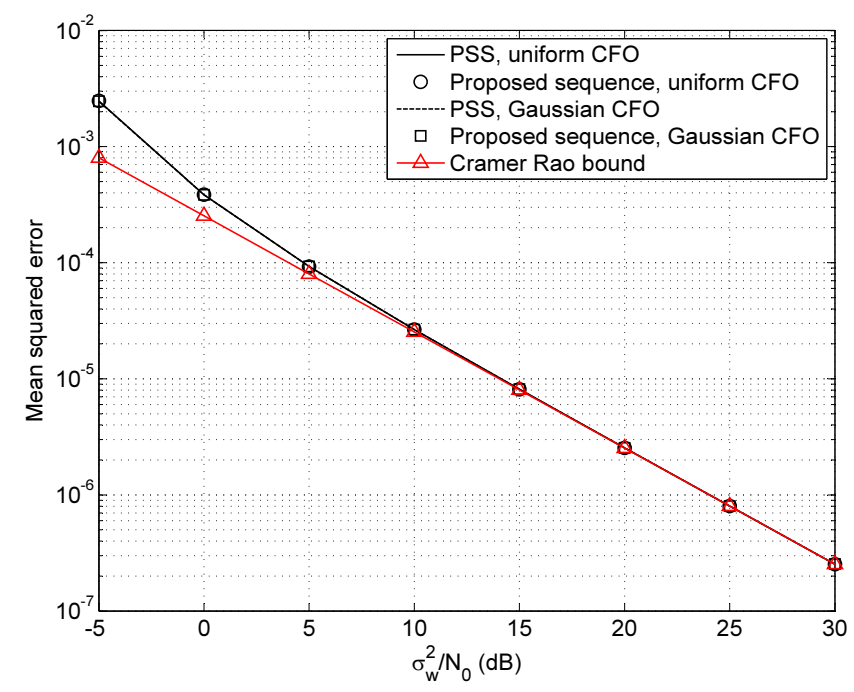

Fig. 4. Single-cell CFO estimation at AWGN channels.

AWGN environments. Now let the channel length $L=4$, the estimation error is shown in Fig. 5. From the figure, the proposed sequence performs nearly the same with the PSS. Therefore in the single-cell environments, the two sequences have comparable estimation accuracy.

Example 4. CFO estimation in multi-cell environments: This example shows the accuracy of CFO estimation for the proposed sequences in multi-cell environments. In this simulation setting, three cells are deployed in the same frequency

$$
\begin{aligned}
q & =\left[\mathbf{d}_{j}^{H} \sum_{i=0}^{I-1} \boldsymbol{\Phi}_{i}^{(1)} \mathbf{F}^{H} \boldsymbol{\Lambda}_{i} \mathbf{w}_{i}\right]^{H}\left[\mathbf{d}_{j}^{H} \sum_{i=0}^{I-1} \boldsymbol{\Phi}_{i}^{(2)} \mathbf{F}^{H} \boldsymbol{\Lambda}_{q} \mathbf{w}_{q}\right] \\
& =[\mathbf{d}_{j}^{H} \boldsymbol{\Phi}_{j}^{(1)} \mathbf{F}^{H} \boldsymbol{\Lambda}_{j} \mathbf{w}_{j}+\sum_{i=0, i \neq j}^{I-1} \underbrace{\mathbf{d}_{j}^{H} \boldsymbol{\Phi}_{i}^{(1)} \mathbf{F}^{H} \boldsymbol{\Lambda}_{i} \mathbf{w}_{i}}_{\mathrm{MCI}_{j \leftarrow i}}]^{H}[\mathbf{d}_{j}^{H} \boldsymbol{\Phi}_{j}^{(2)} \mathbf{F}^{H} \boldsymbol{\Lambda}_{j} \mathbf{w}_{j}+\sum_{i=0, i \neq j}^{I-1} \underbrace{\mathbf{d}_{j}^{H} \boldsymbol{\Phi}_{i}^{(2)} \mathbf{F}^{H} \boldsymbol{\Lambda}_{i} \mathbf{w}_{i}}_{\mathbf{M C I}_{j \leftarrow i}}] .
\end{aligned}
$$






Fig. 5. Single-cell CFO estimation at multipath length $L=4$.

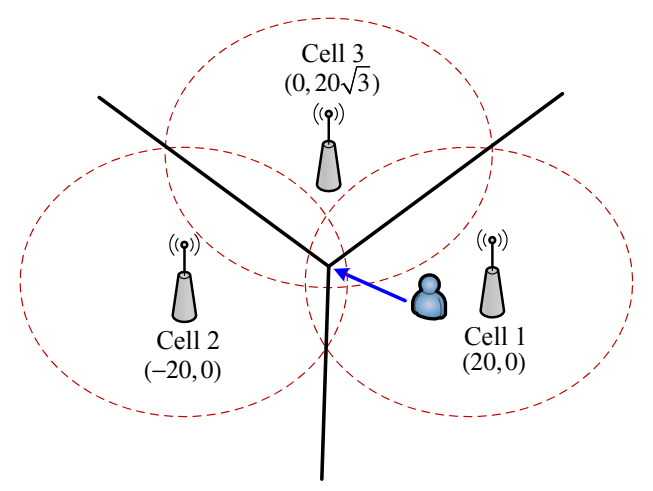

Fig. 6. A multi-cell scenario.

band and its layout is shown in Fig. 6. User is located at different locations, i.e., Location $1=(18,0)$ and Location $3=(0,20 / \sqrt{3})$, for comparing the estimation accuracy between the PSS and the proposed sequences. The path loss model in this example is $38.5+20 \log (r) \mathrm{dB}$ [30]. Also, we consider both uniform and Gaussian CFO scenarios like that in Example 2. The column indices of the proposed sequences are $\{0,4,8\}$ chosen from $\mathbf{W}$. The channel length $L$ is 4 .

The estimation accuracy between the proposed sequence and the PSS is shown in Fig. 7 for the user at Location 1, and in Fig. 8 at Location 3. Note that Location 3 is the boundary region of the three cells. Therefore the receiver needs to perform cell detection before CFO estimation.

From the simulation results we see that the estimation accuracy of the proposed sequence is much better than that of the PSS at both locations. The improved accuracy is due to that the proposed sequence can completely eliminate the dominant MCI arisen from the CFO, and only leave certain ignorable residual MCI. Moreover, we see from the figure that the performance improvement is more pronounced when the user is at the boundary region of different cells. In this case, the induced MCI is much stronger. Thus, using the proposed sequences can better overcome the MCI issue.

Example 5. Performance of linking to a cell: Using the

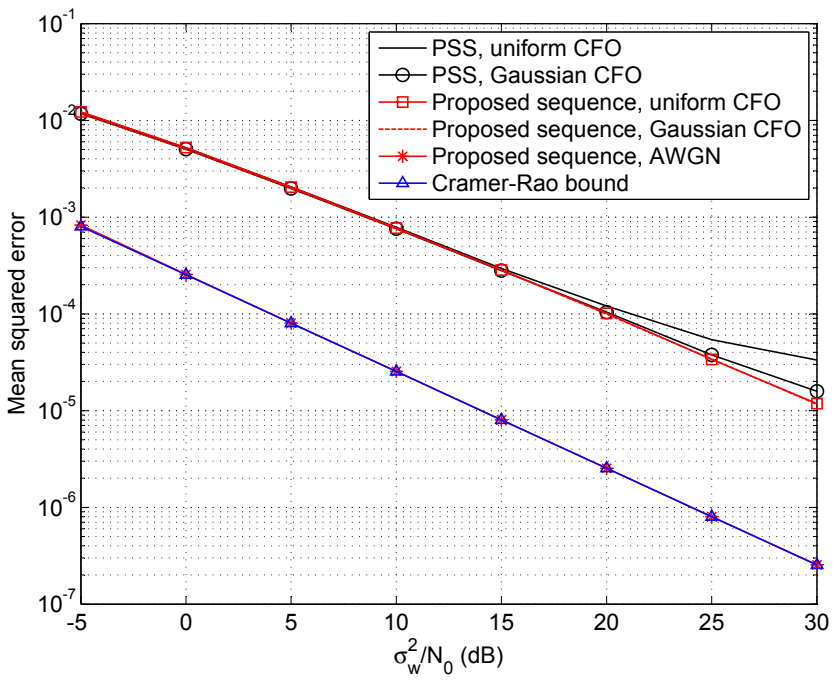

Fig. 7. Multi-cell CFO estimation at Location 1.

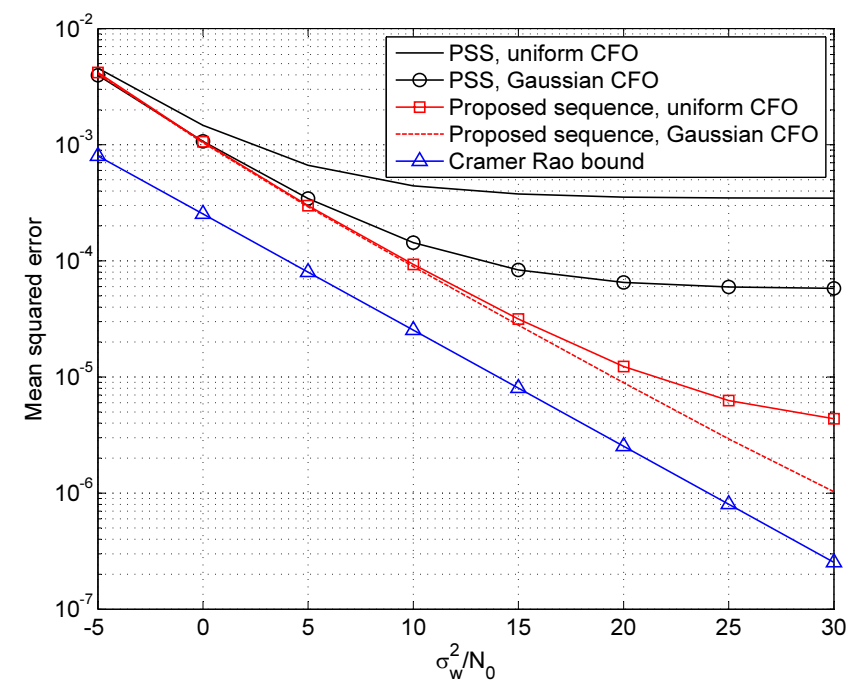

Fig. 8. Multi-cell CFO estimation at Location 3.

same parameter setting as in Example 4, The CFO is normally distributed with outage probability $\operatorname{Pr}(|\mathrm{CFO}|>0.1)=10^{-5}$. Without losing generality, let Cell 1 be the targeted cell to link. Fig. 9 shows the performance of linking in terms of detection error rate, i.e., the probability that the user fails linking to the targeted cell. Observe that the proposed sequences have better linking performance than the PSS applied in the LTE standard under multipath channels. This is a reasonable result because the proposed sequences do not have MCI under multipath environments.

\section{CONCLUSION}

In this paper we proposed sequences for multi-cell environments. We theoretically proved that the proposed sequences have good properties including constant amplitude, zero autocorrelation, and orthogonality in multipath channel environments. Moreover, we analyzed the cyclic shift property of the proposed sequences and proposed a fast algorithm to identify which sequences are the cyclically shifted version of the 


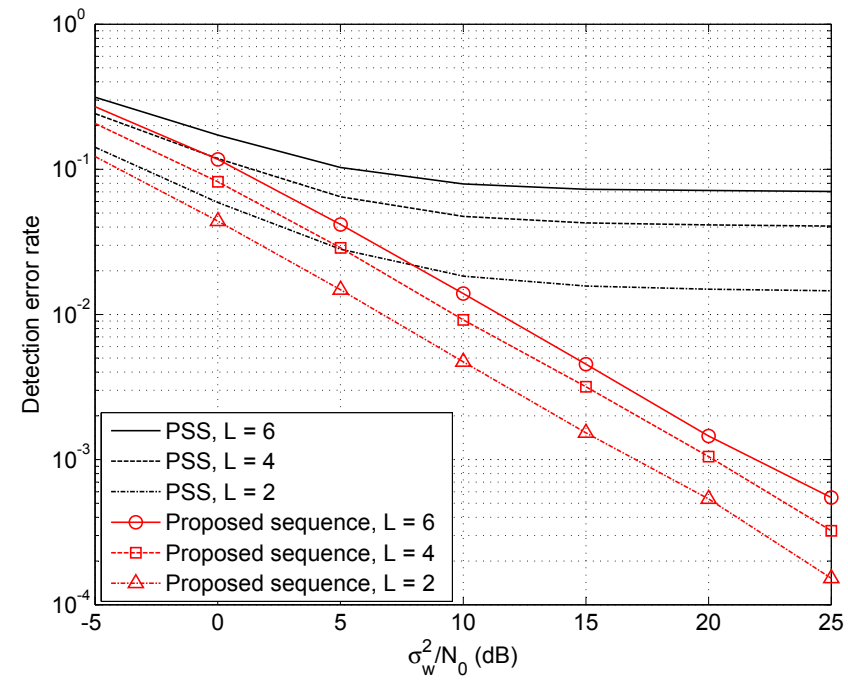

Fig. 9. Performance of linking for the PSS and the proposed sequences.

others, so that the proposed sequences can be used to estimate integer CFO. Furthermore, we analyzed the induced MCI of the proposed sequences when there are fractional CFOs. The analytical results showed that the proposed sequence can completely eliminate the dominate MCI, and only leave the residual MCI, which is in general negligible. Hence, we can slightly modify the CFO estimation algorithms designed for singleuser or single-cell environments and applied them in multi-cell environments. We took a popular CFO estimation for instance, modified and applied it in multi-cell environments. Finally, simulation results showed the analytical results are accurate, and the proposed sequences and CFO estimation algorithms outperform conventional schemes in multi-cell environments. The performance improvement of the proposed sequences is more pronounced when the users is at the boundary regions of several cells, because serious MCI appears in this case. We conclude that with low computational complexity, the proposed sequences and algorithms can efficiently overcome the MCI issue in multi-cell networks.

\section{APPENDIX}

\section{A. Proof of Lemma 1}

If $N$ is even, from (6), $x\left[((k-i))_{N}\right]$ is expressed as

$$
\begin{aligned}
x\left[((k-i))_{N}\right] & =x[k-i+c N]=e^{-j \frac{\pi}{N} u_{1}(k-i+c N)^{2}} \\
& =e^{-j \frac{\pi}{N}\left[u_{1}(k-i+)^{2}+2 c N u_{1}(k-i)+u_{1} c^{2} N^{2}\right]} \\
& =x[k-i] e^{-j 2 \pi u_{1} c(k-i)} e^{-j \pi u_{1} c^{2} N} \\
& =x[k-i] e^{-j \pi u_{1} c^{2} N},
\end{aligned}
$$

where $c \in Z$. Since $N$ is even, $e^{-j \pi u_{1} c^{2} N}=1$. Therefore, $x\left[((k-i))_{N}\right]=x[k-i]$.
If $N$ is odd, from (6), $x\left[((k-i))_{N}\right]$ can be expressed as

$$
\begin{aligned}
& x\left[((k-i))_{N}\right]=x[k-i+c N] \\
& =e^{-j \frac{\pi}{N} u_{1}(k-i+c N)(k-i+c N+1)} \\
& =e^{-j \frac{\pi}{N}\left[u_{1}(k-i)(k-i+1)+2 c N u_{1}(k-i)+u_{1} c N(c N+1)\right]} \\
& =x[k-i] e^{-j 2 \pi u_{1} c(k-i)} e^{-j \pi u_{1} c(c N+1)} \\
& =x[k-i] e^{-j \pi u_{1} c(c N+1)},
\end{aligned}
$$

where $c \in Z$. Since $N$ is odd, $e^{-j \pi u_{1} c(c N+1)}=1$. Therefore, $x\left[((k-i))_{N}\right]=x[k-i]$.

\section{B. Proof of Lemma 2}

According to (5), $w_{i}\left[((k-m))_{N}\right]$ is expressed as.

$w_{i}\left[((k-m))_{N}\right]=w_{i}[k-m+c N]=e^{-j \frac{\pi}{N} \alpha_{(k-m+c N) i}}, c \in \mathbb{Z}$.

If $N$ is even, $\alpha_{(k-m+c N) i}$ can be expressed as (58), shown at the top of the next page. Therefore,

$$
\begin{aligned}
& w_{i}\left[((k-m))_{N}\right] \\
& =e^{-j \frac{\pi}{N} \alpha_{(k-m) i}} e^{-j 2 \pi c Y_{1}(k, m, i)} e^{-j \pi c^{2} N\left(u_{1}+u_{2}\right)} \\
& =w_{i}[k-m] e^{-j \pi c^{2} N\left(u_{1}+u_{2}\right)} .
\end{aligned}
$$

Since $N$ is even, $e^{-j \pi c^{2} N\left(u_{1}+u_{2}\right)}=1$ and $w_{i}\left[((k-m))_{N}\right]=$ $w_{i}[k-m]$.

If $N$ is odd, $\alpha_{(k-m+c N) i}$ is expressed as (59), shown at the top of the next page. Therefore,

$$
\begin{aligned}
& w_{i}\left[((k-m))_{N}\right] \\
& =e^{-j \frac{\pi}{N} \alpha_{(k-m) i}} e^{-j 2 \pi c Y_{2}(k, m, i)} e^{-j \pi c(c N+1)\left(u_{1}+u_{2}\right)} \\
& =w_{i}[k-m] e^{-j \pi c(c N+1)\left(u_{1}+u_{2}\right)} .
\end{aligned}
$$

Since $N$ is odd, $e^{-j \pi c(c N+1)\left(u_{1}+u_{2}\right)}=1$ and $w_{i}[((k-$ $\left.m))_{N}\right]=w_{i}[k-m]$.

\section{ACKNOWLEDGMENT}

The authors would like to thank all the anonymous reviewers for their constructive suggestions, which have significantly improved the quality of this work.

\section{REFERENCES}

[1] 3GPP TS36.211 v8.9.0, Physical Channels and Modulation (Release 8), Dec. 2009.

[2] V. Chandrasekhar, J. G. Andrews, and A. Gatherer, "Femtocells networks: a survey," IEEE Commun. Mag., vol. 46, no. 9, pp. 59-67, Sept. 2008.

[3] A. Attar, V. Krishnamurthy, and O. N. Gharehshiran, "Interference management using cognitive base station for UMTS LTE," IEEE Commun. Mag., vol. 49, no. 8, pp. 152-159, Aug. 2011.

[4] J. G. Andrews, W. Choi, and R. W. Heath Jr., "Overcoming interference in spatial multiplexing MIMO cellular networks," IEEE Wireless Commun., vol. 14, no. 6, pp. 95-104, Dec. 2007.

[5] S. Shim, J. S. Kwak, R. W. Heath, Jr., and J. G. Andrews, "Block diagonalization for multi-user MIMO with other-cell interference," IEEE Trans. Wireless Commun., vol. 7, no. 7, pp. 2671-2681, July 2008.

[6] J. Zhang and J. G. Andrews, "Adaptive spatial intercell interference cancellation in multicell wireless networks," IEEE J. Sel. Areas Commun., vol. 28, no. 9, pp. 1455-1468, Dec. 2010.

[7] A. Nosratinia, T. E. Hunter, and A. Hedayat, "Cooperative communication in wireless networks," IEEE Commun. Mag., vol. 42, no. 10, pp. 74-80, Oct. 2004

[8] S. Jagannathan, H. Aghajan, and A. Goldsmith, "The effect of time synchronization errors on the performance of cooperative MISO systems," in Proc. 2004 IEEE Globecom, pp. 102-107. 


$$
\begin{aligned}
\alpha_{(k-m+c N) i} & =u_{1}(k-m+c N-i)^{2}+u_{2}(k-m+c N)^{2} \\
& =u_{1}(k-m-i)^{2}+u_{2}(k-m)^{2}+u_{1}[2 c N(k-m-i)]+u_{2}[2 c N(k-m)]+u_{1} c^{2} N^{2}+u_{2} c^{2} N^{2} \\
& =\alpha_{(k-m) i}+2 c N \underbrace{\left[u_{1}(k-m-i)+u_{2}(k-m)\right]}_{Y_{1}(k, m, i)}+\left(u_{1}+u_{2}\right) c^{2} N^{2}
\end{aligned}
$$

$$
\begin{aligned}
\alpha_{(k-m+c N) i} & =u_{1}(k-m+c N-i)(k-m+c N-i+1)+u_{2}(k-m+c N)(k-m+c N+1) \\
& =u_{1}(k-m-i)(k-m-i+1)+u_{2}(k-m)(k-m+1)+u_{1}[2 c N(k-m-i)]+u_{2}[2 c N(k-m)] \\
& +u_{1} c N(c N+1)+u_{2} c N(c N+1) \\
& =\alpha_{(k-m) i}+2 c N \underbrace{\left[u_{1}(k-m-i)+u_{2}(k-m)\right]}_{Y_{2}(k, m, i)}+c N(c N+1)\left(u_{1}+u_{2}\right)
\end{aligned}
$$

[9] Q. Huang, M. Ghogho, J. Wei, and P. Ciblat, "Practical timing and frequency synchronization for OFDM-based cooperative systems," IEEE Trans. Signal Process., vol. 58, no. 7, pp. 3706-3716, July 2010.

[10] V. Chandrasekhar and J. G. Andrews, "Spectrum allocation in tiered cellular networks," IEEE Trans. Wireless Commun., vol. 57, no. 10, pp. 3059-3068, Oct. 2009.

[11] V. Chandrasekhar, J. G. Andrews, T. Muharemovic, Z. Shen, and A. Gatherer, "Power control in two-tier femtocell networks," IEEE Trans. Wireless Commun., vol. 8, no. 8, pp. 4316-4328, Aug. 2009.

[12] C. U. Saraydar, N. B Mandayam, and D. J. Goodman, "Pricing and power control in a multicell wireless data network," IEEE J. Sel. Areas Commun., vol. 19, no. 10, pp. 1883-1892, Oct. 2001.

[13] D. Gesbert, S. Kiani, and A. Gjendemsj, "Adaptation, coordination, and distributed resource allocation in interference-limited wireless networks," Proc. IEEE, vol. 95, no. 12, pp. 2393-2409, Dec. 2007.

[14] B. M. Popovic, "Generalized chirp-like polyphase sequence with optimum correlation properties," IEEE Trans. Inf. Theory, vol. 38, no. 4, pp. 1406-1409, July 1992.

[15] S.-H. Tsai, Y.-P. Lin, and C.-C. J. Kuo, "MAI-free MC-CDMA systems based on Hadamard Walsh codes," IEEE Trans. Signal Process., vol. 54, no. 8, pp. 3166-3179, Aug. 2006.

[16] L. Tadjpour, S.-H. Tsai, and C.-C. J.Kuo, "Simplified multiaccess interference reduction for MC-CDMA with carrier frequency offset," IEEE Trans. Veh. Technol., vol. 59, no. 5, pp. 2543-2555, June 2010.

[17] L. Sanguinetti, L. Taponecco, and M. Morelli, "Interference-free code design for MC-CDMA uplink transmissions," IEEE Trans. Wireless Commun., vol. 8, no. 11, pp. 5461-5465, 2009.

[18] P. H. Moose, "A technique for orthogonal frequency division multiplexing frequency offset correction," IEEE Trans. Commun., vol. 42, no. 10, pp. 2908-2914, Oct. 1994.

[19] T. M. Schmidl and D. C. Cox, "Robust frequency and timing synchronization for OFDM," IEEE Trans. Commun., vol. 45, no. 12, pp. 16131621, Dec. 1997.

[20] J.-J. van de Beek, M. Sandell, and P. O. Borjesson, "ML estimation of timing and frequency offset in OFDM systems," IEEE Trans. Signal Process., vol. 45, no. 7, pp. 1800-1805, July 1997.

[21] LG Electronics, "Comparison of sequence and structure for P-SCH," 3GPP Tech. Doc., Tdoc R1-071531, St. Julians, Malta 26 - 30, Mar. 2007.

[22] I. Kim, Y. Han, Y. Kim, and S. C. Bang, "Sequence hopping cell search scheme for OFDM cellular systems," IEEE Trans. Wireless Commun., vol. 7, no. 5, pp. 1483-1489, May 2008.

[23] A. Roessler, "Cell search and cell selection in UMTs LTE," Rohde and Schwarz Technology Report, Sept. 2009.

[24] K. Manolakis, D. M. Gutierrez Estevez, V. Jungnickel, X. Wen, and C. Drewes, "A closed concept for synchronization and cell search in 3GPP LTE systems," in Proc. 2009 IEEE WCNC, pp. 1-6.

[25] Y. Tsai, G. Zhang, D. Grieco, F. Ozluturk, and X. Wang "Cell search in 3GPP long term evolution systems," IEEE Veh. Technol. Mag., vol. 2, no. 2, pp. 23-29, June 2007.

[26] M. Moretti, A. Todini, A. Baiocchi, and G. Dainelli, "A layered architecture for fair resource allocation in multicellular multi-carrier systems," IEEE Trans. Veh. Technol., vol. 60, no. 4, pp. 1788-1798, Apr. 2011.
[27] M. Morelli and M. Moretti, "Carrier frequency offset estimation for OFDM Direct-Conversion receivers," IEEE Trans. Wireless Commun., vol. 11, no. 7, pp. 2670-2679, July 2012.

[28] K. Lee, S.-H. Moon, S. Kim, and I. Lee, "Sequence designs for robust consistent frequency-offset estimation in OFDM systems," IEEE Trans. Veh. Technol., vol. 62, no. 3, pp. 1389-1394, Mar. 2013.

[29] T. Andreescu, D. Andrica, and I. Cucurezeanu, An Introduction to Diophantine Equations: A Problem-Based Approach. Springer, 2010.

[30] H. Claussen, L. T. W. Ho, and L. G. Samuel, "An overview of the femtocell concept," Bell Labs Technical J., vol. 13, no. 1, pp. 221-245, Mar. 2008.

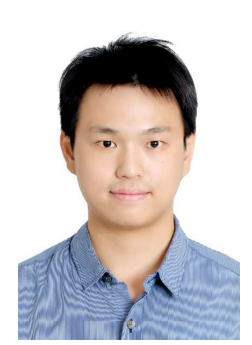

Ying-Che Hung was born in Taichung, Taiwan, in 1983. He received the B.S. degree in Power Mechanical Engineering from the National Tsing-Hua University, Taiwan, in 2006 . He is currently pursuing the Ph.D. degree with the Department of Electrical Engineering, National Chiao-Tung University, Taiwan. His research interests mainly include digital signal processing, multiple-input-multiple-output (MIMO) wireless communications, and compressive sensing.

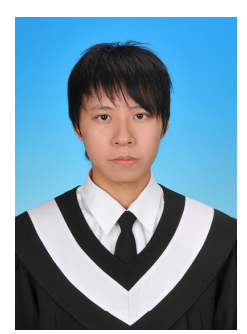

Sheng-Yuan Peng was born in Taipei, Taiwan, in 1988. He received the B.S. degree in Electronic and Computer Engineering from the National Taiwan University of Science and Technology, Taiwan, in 2010 and the M.S. degree in Communications Engineering from National Chiao-Tung University, Taiwan, in 2012. His research interests include signal processing for wireless communications and multiple-input-multiple-output (MIMO) systems.

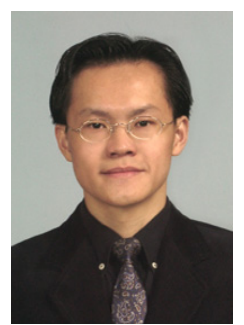

Shang-Ho (Lawrence) Tsai (SM'12) was born in Kaohsiung, Taiwan, 1973. He received the Ph.D. degree in Electrical Engineering from the University of Southern California (USC), USA, in Aug. 2005. From June 1999 to July 2002, he was with the Silicon Integrated Systems Corp. (SiS), where he participated the VLSI design for DMT-ADSL systems. From Sep. 2005 to Jan. 2007, he was with the MediaTek Inc. (MTK) and participated the VLSI design for MIMO-OFDM systems. From Jun. 2013 to Dec. 2013, he was a visiting fellow in the department of Electrical Engineering at the Princeton University. Since Feb. 2007, he joined the Department of Electrical and Control Engineering (now Department of Electrical Engineering) at the National Chiao Tung University where he is now an associate professor. His research interests include signal processing for communications, statistical signal processing, and signal processing for VLSI designs. He was awarded a government scholarship for overseas study from the Ministry of Education, Taiwan, in 2002-2005. 